\title{
Are the Formation and Abundances of Metal-poor Stars the Result of Dust Dynamics?
}

\author{
Philip F. Hopkins ${ }^{1}$ and Charlie Conroy ${ }^{2}$ \\ ${ }^{1}$ TAPIR, Mailcode 350-17, California Institute of Technology, Pasadena, CA 91125, USA; phopkins@ caltech.edu \\ ${ }^{2}$ Harvard-Smithsonian Center for Astrophysics, 60 Garden Street, Cambridge, MA 02138, USA \\ Received 2015 December 11; revised 2016 October 8; accepted 2016 October 10; published 2017 January 24
}

\begin{abstract}
Large dust grains can fluctuate dramatically in their local density, relative to the gas, in neutral turbulent disks. Small, high-redshift galaxies (before reionization) represent ideal environments for this process. We show via simple arguments and simulations that order-of-magnitude fluctuations are expected in local abundances of large grains $(>100 \AA)$ under these conditions. This can have important consequences for star formation and stellar metal abundances in extremely metal-poor stars. Low-mass stars can form in dust-enhanced regions almost immediately after some dust forms even if the galaxy-average metallicity is too low for fragmentation to occur. We argue that the metal abundances of these "promoted" stars may contain interesting signatures as the $\mathrm{CNO}$ abundances (concentrated in large carbonaceous grains and ices) and $\mathrm{Mg}$ and Si (in large silicate grains) can be enhanced and/or fluctuate almost independently. Remarkably, the otherwise puzzling abundance patterns of some metal-poor stars can be well fit by standard IMF-averaged core-collapse $\mathrm{SNe}$ yields if we allow for fluctuating local dust-to-gas ratios. We also show that the observed log-normal distribution of enhancements in these species agrees with our simulations. Moreover, we confirm that $\mathrm{Mg}$ and $\mathrm{Si}$ are correlated in these stars; the abundance ratios are similar to those in local silicate grains. Meanwhile $[\mathrm{Mg} / \mathrm{Ca}]$, predicted to be nearly invariant from pure SNe yields, shows very large enhancements and variations up to factors of $\gtrsim 100$ as expected in the dust-promoted model, preferentially in the [C/Fe]-enhanced metal-poor stars. Together, this suggests that (1) dust exists in second-generation star formation, (2) local dust-to-gas ratio fluctuations occur in protogalaxies and can be important for star formation, and (3) the light element abundances of these stars may be affected by the local chemistry of dust where they formed, rather than directly tracing nucleosynthesis from earlier populations.
\end{abstract}

Key words: dark ages, reionization, first stars - galaxies: formation - galaxies: star formation - stars: abundances stars: formation - turbulence

\section{Introduction}

Extremely metal-poor stars represent a laboratory for studying the conditions in the early universe. They are sensitive probes of stellar evolution and supernovae (SNe) explosion models, nucleosynthesis and the origin of elements heavier than $\mathrm{H}$ and $\mathrm{He}$, the enrichment and early formation history of galaxies, and the nature and origins of the first stars. Their metal abundance patterns present many outstanding challenges and unsolved problems; understanding the origin of these stars and their stellar abundances is critical to all of the open questions above.

For example, most of the observed extremely metal-poor $([\mathrm{Fe} / \mathrm{H}]<-3.0)$ population appears to be dramatically enhanced in light elements (CNO) ( $>40 \%$ and $>80 \%$ of stars below $[\mathrm{Fe} / \mathrm{H}]<-3.0$ and -4.0 ; Placco et al. 2014), including the various subclasses of carbon-enhanced metal-poor stars (CEMPS) with $[\mathrm{C} / \mathrm{Fe}] \sim 0-4$ (Lee et al. 2013). A plausible explanation for the carbon enhancements is essentially a selection effect: lowmass star formation requires efficient cooling (to allow collapse and fragmentation); clouds with a total metal mass that is too low $([Z / H] \ll-3)$ would either form stars very inefficiently, or only form massive (short-lived) stars (Schneider et al. 2003; Omukai et al. 2005; Chiaki et al. 2014; Ji et al. 2014), so stars selected with very low $[\mathrm{Fe} / \mathrm{H}]$ "should," in this argument, have high abundances in the CNO species constituting most of the metal mass. ${ }^{3}$ But this

\footnotetext{
3 In this paper, we generally use "CEMP" as a shorthand for CNO-enhanced, extremely metal-poor stars, including NEMP and OEMP stars, which are believed to form early in the universe and with metal abundances reflecting their formation conditions rather than stellar evolution or pollution by a binary companion. This means that we focus on the CEMP-no population, dominant at $[\mathrm{Fe} / \mathrm{H}]<-3$, rather than the CEMP-r, CEMP-s, and CEMP-r/s populations (for extended discussion, see Carollo et al. 2014; Bonifacio et al. 2015; Hansen et al. 2015; Maeder et al. 2015).
}

only explains why we might preferentially see CEMPS today, not how the enhancement (e.g., high $[\mathrm{C} / \mathrm{Fe}]$ ) was produced in the first place. And it does not necessarily tell us anything about the galaxies or environments in which these stars formed, only about the local cloud that collapsed to form the star. Other light elements such as $\mathrm{Si}$ and $\mathrm{Mg}$ also display unusual enhancements and correlations that are not well understood (see, e.g., Aoki et al. 2002); in ultra-faint dwarfs these elements have also been observed to vary and usually appear in excess together (Aoki et al. 2002; Norris et al. 2010a), but in limited cases may also vary independently (Norris et al. 2010b; Frebel et al. 2014).

The simplest explanation for these metal abundances, i.e., that they reflect the yields of normal core-collapse $\mathrm{SNe}$ (averaged over the stellar initial mass function (IMF)), fails to predict anything like the observed stellar abundances of the extremely metal-poor stars $([\mathrm{Fe} / \mathrm{H}]<-3)$. Of course, at these low metallicities, the number of progenitor $\mathrm{SNe}$ enriching the ISM may be small, so models typically allow for metal-poor or metal-free progenitor stars, and an arbitrary mix of progenitor stellar masses (i.e., assuming the abundances might come from just one or at most a few $\mathrm{SNe}$ with individual explosion and progenitor parameters fitted to the observations). However, even with these degrees of freedom, the models still often fail to explain the abundances of certain individual species at the order-of-magnitude level (see, e.g., Nomoto et al. 2006; Heger \& Woosley 2010; Lee et al. 2014; Placco et al. 2015), although they undoubtedly explain many of the observed abundance ratios. For the lowest metallicity stars observed $([\mathrm{Fe} / \mathrm{H}]<-4)$, and in particular for the CEMP stars, these remaining discrepancies have led to more "exotic" 
models with a number of free parameters, invoking a mix of normal/faint $\mathrm{SNe}$ and hypernovae (with variable explosion energies of $\sim 10^{51}-10^{54}$ erg); jets, prior "failed explosions," and fallback episodes; rotation and adjustable mixing layers allowing for a tunable stellar abundance profile in the progenitor stars; and pollution of the stars via companions (e.g., Tominaga et al. 2007; Ishigaki et al. 2014; Takahashi et al. 2014; Abate et al. 2015). These additions can improve the agreement with observations; however, there is still no consistent theoretical scenario that simultaneously explains most of the observed stars, and even the best-fit models for many individual stars can still have order-of-magnitude discrepancies with certain outlier elements (see Tominaga et al. 2014; Frebel et al. 2015; Placco et al. 2015, and references therein).

An alternative explanation, therefore, which has thus far not been much discussed (although see Gilmore et al. 2013; Maeder et al. 2015), is that the metallicities (and even abundance ratios) of regions that successfully form low-mass stars at extremely low metallicities do not necessarily reflect the direct $\mathrm{SNe}$ yields. This can happen via many distinct physical mechanisms. One mechanism, which is particularly appealin$\mathrm{g}$ and already known to occur under the right conditions, is the segregation of dust and gas by aerodynamic drag. In short, in primarily neutral dense gas, massive dust grains (which, at least at low redshift, contain a large fraction of all the ISM metal mass) behave like aerodynamic particles (i.e., they are coupled to the gas via drag from collisions with atoms or molecules). As such, they do not move perfectly with gas, and under certain circumstances they can clump or disperse independently of the gas, generating large fluctuations in the dust-to-gas ratio. By extension, this leads to fluctuations in the abundance of different species (those concentrated in grains of the correct size) from one region to another.

In protoplanetary disks, this phenomenon has been wellstudied and is believed to be critical for planetesimal formation. When stirred by turbulence, trapped in long-lived vortices or pressure extrema (vortex or pressure traps), or excited by motions generated by the dust-gas interaction itself (i.e., the streaming instability), the local number density of dust grains can fluctuate by several orders of magnitude relative to gas (see, e.g., Bracco et al. 1999; Cuzzi et al. 2001; Youdin \& Goodman 2005; Johansen \& Youdin 2007; Bai \& Stone 2010c; Hopkins 2016b). The same phenomenon of "preferential concentration" is well known in both laboratory experiments and simulations of terrestrial turbulence (Squires \& Eaton 1991; Rouson \& Eaton 2001; Monchaux et al. 2010, 2012; Pan et al. 2011). Because the dynamics are scale-free, the same phenomena governing pebbles and boulders in a protoplanetary disk should apply to submicron size dust in a giant molecular cloud (Padoan et al. 2006; Yoshimoto \& Goto 2007; Bec et al. 2009; Olla 2010; Hopkins 2016a). And indeed, dust-to-gas ratio fluctuations on scales of $\sim 0.003-10 \mathrm{pc}$ have been measured in many nearby molecular clouds and in some galaxy nuclei (e.g., Orion, Taurus, and many more; see Thoraval et al. 1997, 1999; Abergel et al. 2002; MivilleDeschênes et al. 2002; Padoan et al. 2006; Flagey et al. 2009; Pineda et al. 2010; Nyland et al. 2013); the dependence on dust grain size is in good agreement with the predictions of turbulent concentration (Padoan et al. 2006; Hopkins 2014). As a result, Hopkins (2014) speculated that these might lead to local variations in the metallicity of star-forming regions and metal abundances of the resulting stars. However, they concluded that, in the Local Universe, the $\sim 1 \sigma$ scatter in metal abundances due to these effects would be $<0.1 \mathrm{dex}$ in most cases; order-of-magnitude effects would be extremely rare.

In this paper, we consider instead how such fluctuations in the local dust-to-gas ratio might occur in high-redshift, predominantly neutral galaxies. Using both simulations and analytic arguments, we show that the effects of turbulent concentration may be much more dramatic in these protogalaxies, potentially leading to large fluctuations in the local metallicity that can generate locally dust-rich and metal-rich regions that will preferentially cool, fragment, and form lowmass stars (i.e., "promoted" star formation). Using a simple model for dust, we examine how this might alter the interpretation of the observed metal abundances of the stars. Remarkably, we show that some of the light-element (CNO, $\mathrm{Mg}$, and $\mathrm{Si}$ ) enhancements and correlations that are very difficult to reproduce in $\mathrm{SNe}$ nucleosynthesis models result naturally from the assumption that stars formed in unusually dust-rich regions.

\section{The Scenario}

Before going into the details, we briefly sketch the scenario we will explore. In a high-redshift mini-halo, one or more PopIII $\mathrm{SNe}$ explode, providing a seed amount of dust $(-7 \lesssim$ $[Z / H] \lesssim-3)$. That initial dust-to-gas ratio can then, under the right conditions, be enhanced by one or more orders of magnitude. The enhanced regions will then have local metallicities high enough for standard cooling, fragmentation, and low-mass star formation to occur at high densities. Any low-mass (long-lived) stars that form this way will be imprinted with elemental abundances reflecting the unusually large dust concentrations of their formation sites.

Conditions for this process are very favorable in the gas disks of high-redshift galaxies because they are primarily neutral (so collisional drag rather than Coulomb and Lorentz forces dominates dust dynamics, and grain ices are not suppressed by the radiation field) and because they are metalpoor, so that under mean conditions cooling and star formation (at least low-mass star formation) are inefficient. The latter means that the surviving low-mass relics of this era will be biased toward the products of the dust-concentration process we describe, even if the conditions required are rare.

\subsection{Initial Conditions}

Consider a (mini)-halo at pre-reionization redshifts $(z \gtrsim 6)$ tha$t$ recently formed a first generation of stars. Such halos are predicted to contain a gaseous disk of mostly neutral gas (free electron fractions $f_{\text {ion }} \sim 10^{-6}-10^{-2}$ ), to be rapidly polluted by trace metallicity (dispersed by $\mathrm{SNe}$ ), and to be turbulent with transonic Mach numbers $\left(\mathcal{M} \equiv v_{\text {turb }} / c_{s} \sim 1\right.$ owing to, e.g., galactic rotation, accretion, and gravitational instability); (see, e.g., Greif et al. 2008; Wise et al. 2008; Wise \& Abel 2008; Muratov et al. 2013; Pawlik et al. 2013).

Let us make the ansatz that a non-negligible fraction of the metal mass $\left(\langle Z\rangle \equiv M_{\text {metal }} / M_{\text {gas }}=\left\langle\rho_{\text {metals }}\right\rangle /\left\langle\rho_{\text {gas }}\right\rangle\right)$ is in dust $\left(Z_{d} \equiv \rho_{\text {dust }} / \rho_{\text {gas }}\right)$. In both observations and theory (e.g., Mathis et al. 1977; Li \& Draine 2001; Draine 2003; Gordon et al. 2003; Draine \& Li 2007; De Marchi \& Panagia 2014), most of the grain mass is always in the largest grains, for which we define the size $a \equiv a_{\mu} \mu \mathrm{m}$ (typically $a_{\mu} \sim 0.1-10$; Grun 
et al. 1993; Landgraf et al. 2000; Witt et al. 2001; Altobelli et al. 2007; Goldsmith et al. 2008; Poppe et al. 2010; Schnee et al. 2014).

\subsection{Dust-to-gas Fluctuations: Physics}

Large ( $>\AA$-sized) dust grains do not form a fluid, but behave (in neutral gas) like aerodynamic particles ${ }^{4}$ that feel a drag: $D v_{\text {dust }} / D t=-\left(v_{\text {dust }}-v_{\text {gas }}\right) / t_{\mathrm{s}}$; here the drag or stopping time is written

$$
t_{\mathrm{s}} \equiv \frac{\bar{\rho}_{\text {solid }} a}{\rho_{\mathrm{g}} c_{s}} \approx 1.6 \times 10^{7} \text { year }\left(\frac{n_{\mathrm{gas}}}{\mathrm{cm}^{-3}}\right)^{-1}\left(\frac{T_{\mathrm{gas}}}{1000 \mathrm{~K}}\right)^{-1 / 2} a_{\mu}
$$

where $\bar{\rho}_{\text {solid }} \approx 2.4 \mathrm{~g} \mathrm{~cm}^{-3}$ is the internal grain density (Weingartner \& Draine 2001), and $\rho_{\mathrm{g}}$ and $c_{s}$ are the gas density and sound speed.

Because of this partial coupling, grains can experience large, coherent density fluctuations relative to the gas. This occurs on spatial scales $\sim R$ where there are velocity structures (e.g., turbulent eddies) with characteristic timescales resonant with $t_{\mathrm{s}}$ (i.e., $t_{\mathrm{s}} \sim\left|t_{e}(R)\right| \equiv R /\left\langle v_{t}^{2}(R)\right\rangle^{1 / 2}$; Cuzzi et al. 2001; Hogan \& Cuzzi 2007; Bec et al. 2009; Olla 2010; Hopkins 2016a). If $\left|t_{e}\right| \ll t_{\mathrm{s}}$, grains simply pass through structures without significant perturbation; if $\left|t_{e}\right| \gg t_{\mathrm{s}}$, grains are well entrained (i.e., move with the gas). In a rotating disk, the most dramatic fluctuations appear when $t_{\mathrm{s}} \sim \Omega^{-1}$ ( $\Omega$ is the orbital frequency, $\Omega\left(r_{\text {disk }}\right)=V_{\mathrm{c}} / r_{\text {disk }} \sim\left(G M_{\text {disk }} / r_{\text {disk }}^{3}\right)^{1 / 2}$; Bracco et al. 1999; Johansen \& Youdin 2007; Carballido et al. 2008; Bai \& Stone 2010b; Dittrich et al. 2013; Jalali 2013; Hopkins 2016b). This scale corresponds to resonance with both the driving scale of turbulence (where most of the power is) and orbital shear (leading to new phenomena like global vortex traps, pressure traps, and zonal flows; see references above). These are well studied in the protoplanetary disk literature, and are believed to play a critical role in planet formation. We therefore define the dimensionless Stokes number as

$$
\begin{aligned}
\tau_{\mathrm{s}} \equiv t_{\mathrm{s}} \Omega & =\frac{2 \bar{\rho}_{\text {solid }} a}{\Sigma_{\text {gas }}} \frac{\sigma}{c_{s}} \\
& \approx 0.23 a_{\mu}\left(1+\mathcal{M}^{2}\right)^{\frac{1}{2}}\left(\frac{10 M_{\odot} \mathrm{pc}^{-2}}{\Sigma_{\text {gas }}}\right) .
\end{aligned}
$$

Here $\Sigma_{\text {gas }} \approx 2\left\langle\rho_{\mathrm{g}}\right\rangle H$ is the gas disk surface density $\left(H=\sigma \Omega^{-1}\right.$ is its scale height, with $\left.\sigma^{2}=c_{s}^{2}+v_{\text {turb }}^{2}(H)\right)$.

A value of $\tau_{\mathrm{s}} \sim 1$ predicts maximum fluctuations in the dustto-gas ratio on large $\sim H$ scales. Several groups have studied a case very similar to the one of interest here, $\tau_{\mathrm{s}} \sim 0.01-1$ in a primarily neutral disk with sufficiently low $Z_{d}$ such that

\footnotetext{
4 Following Elmegreen (1979), Draine \& Salpeter (1979), and Draine \& Sutin (1987), we expect dust-dust collisions, Coulomb interactions, and Lorentz forces to be subdominant in the dust momentum equation by factors $\sim Z_{d}\left(1+\tilde{\mathcal{B}} \mathcal{M}^{2}\right)^{-1 / 2} \ll 1, \quad \sim 6 f_{\text {ion }} \ll 1$, and $\sim 0.1 \tilde{B} T_{100 \mathrm{~K}} a_{\mu}^{-1} n_{10}^{-1 / 2} \ll 1$ (where $\tilde{B}$ is the ratio of the magnetic field strength to equipartition, and $\left.n_{10}=n_{\text {gas }} / 10 \mathrm{~cm}^{-3}\right)$, respectively. Even assuming no extinction, radiation pressure only dominates well inside of the Stromgren spheres of massive stars $\left(R_{\mathrm{RP}} / R_{\text {Stromgren }} \approx 0.1\left(n_{10} L_{*} / 10^{4} L_{\odot}\right)^{1 / 6}\right.$, where $R_{\mathrm{RP}}$ is the distance from a star within which radiation pressure dominates over drag). For essentially all reasonable models (discussed in the text), grain formation/destruction timescales in stars are much longer than local gas dynamical times, so do not alter the relevant instabilities. In addition, we assume the Stokes limit for drag, trivially valid for $a \lesssim 10^{13} \mathrm{~cm}$.
}

the "back reaction" (which occurs when $Z_{d} \gg 1$ ) can be neglected. These studies (e.g., Hogan \& Cuzzi 2007; Johansen \& Youdin 2007; Bai \& Stone 2010a; Pan et al. 2011; Dittrich et al. 2013; Hendrix \& Keppens 2014) find an approximately log-normal distribution of dust-to-gas ratios with rms scatter on large $\sim H$ scales of $\sim(0.6,0.9,1.2)$ dex for $\tau_{\mathrm{s}}=(0.1,0.3,1.0) .{ }^{5}$ However, these studies were focused on protoplanetary disks and considered incompressible, slowly cooling (adiabatic) gas with small Mach numbers.

\subsection{Dust-to-gas Fluctuations: Simulations}

In P. F. Hopkins \& H. Lee (2015, in preparation), we consider a suite of simulations of aerodynamic grains in compressible, rapidly cooling (isothermal), magnetized, supersonic turbulence appropriate for galactic disks. For this study, we extend that work by considering the identical setup with a different set of initial conditions (Mach numbers, grain sizes, etc.) chosen to reflect the parameters of interest here. Details of the simulations and extensive discussion of the relevant instabilities are in P. F. Hopkins \& H. Lee (2015, in preparation); for a summary of the specific runs shown here, see the Appendix.

The simulations here are 2D (thin-disk) simulations using the shearing-sheet approximation (i.e., zooming in on a patch of a rotating gas disk in a constant-circular velocity potential), and solve the coupled equations of gravity, magnetohydrodynamics, and grain drag forces (with the full equations valid for compressible gases in both sub- and supersonic limits). They include the effects of the disk gravity, dust drag, hydrodynamics, magnetic fields, and turbulence, which is stirred in the large-eddy approximation to produce quasisteady-state Mach numbers $\mathcal{M} \sim 1-2$. The grain properties are specified by $\bar{\rho}_{\text {solid }} a$ or equivalently the average value $\left\langle\tau_{\mathrm{s}}\right\rangle \equiv \bar{\rho}_{\text {solid }} a \Omega /\left(\left\langle\rho_{\mathrm{g}}\right\rangle\left\langle c_{s}\right\rangle\right)$ (since $\rho$ and $c_{s}$ can vary locally in a simulation).

Figure 1 shows images of the fully developed turbulent dust + gas disks for $\left\langle\tau_{\mathrm{s}}\right\rangle=0.01,0.1,1$. It is obvious upon visual inspection that there are large dust-to-gas ratio fluctuations, and that the characteristic size-scale of fluctuations increases with $\tau_{\mathrm{s}}$. With $\left\langle\tau_{\mathrm{s}}\right\rangle \sim 0.01$, there are large fluctuations from clump to clump (i.e., star forming core to core) within GMClike complexes; with $\left\langle\tau_{\mathrm{s}}\right\rangle \sim 1$, the fluctuations are from complex to complex. Figure 2 quantifies this by plotting the time-averaged distribution of dust-to-gas ratios measured in each simulation. We find, consistent with P. F. Hopkins \& H. Lee (2015, in preparation), a broad distribution of local dust-togas ratios, even in the very high-density gas, with a logarithmic scatter of $\sim 0.3-0.6 \mathrm{dex}$, depending on $\tau_{\mathrm{s}}$ and the local gas density. This implies that fluctuations as large as $\gg 100$ may in fact occur in real galaxies, on spatial scales comparable to or larger than the scales that will collapse to form stars (i.e., dense cores). We show further predictions from these simulations below.

\subsection{Consequences for Star Formation}

In turbulent protogalaxies, self-gravitating atomic/molecular clouds are constantly forming on scales $\sim 0.01-1 H$; even

\footnotetext{
In contrast, for nanometer-scale dust with $\tau_{\mathrm{s}} \sim 10^{-3}$, local fluctuations in the dust-to-gas ratio are predicted to occur, but on scales of $R \sim 10^{-5} \mathrm{H}$, far smaller than the scales of star formation, and so are averaged out on larger scales. Lorentz forces are also likely dominant over collisional drag for these grains.
} 

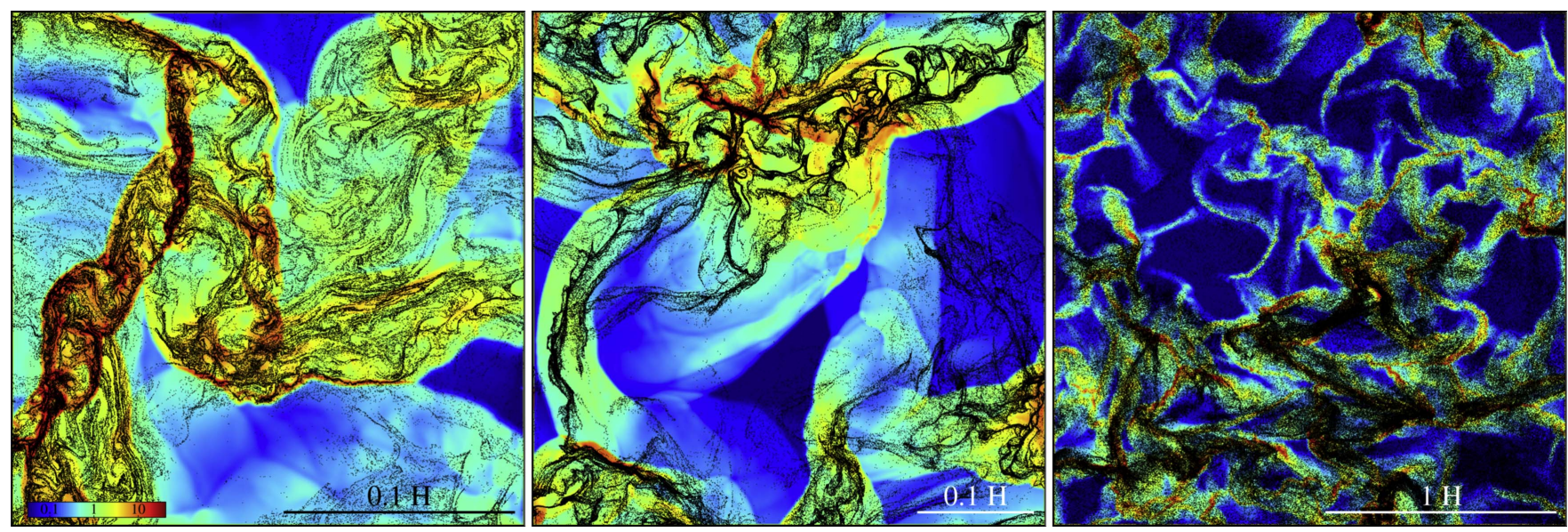

Figure 1. Simulations of aerodynamic dust grains in a shearing, magnetized, turbulent galactic disk, with $\left\langle\tau_{\mathrm{s}}\right\rangle=0.01$ (left), $\left\langle\tau_{\mathrm{s}}\right\rangle=0.1$ (middle), $\left\langle\tau_{\mathrm{s}}\right\rangle=1$ (right). Colors show gas density relative to mean $(\rho /\langle\rho\rangle$; see color bar); black points show dust particles. We show a subvolume of each simulation; scale is shown relative to the disk scale height $H$. The simulations follow grains of a given size including gravitational forces and gas drag, in a transonically MHD-turbulent (Mach numbers $\mathcal{M} \sim 2$ ) isothermal gas disk, representative of the neutral ISM in a high-redshift galaxy. The range of $\tau_{\mathrm{s}}$ corresponds to grain sizes $\sim 0.01-10 \mu \mathrm{m}$, depending on the disk properties (Equation (2)). Dust grains clearly exhibit strong clustering, with large fluctuations in the dust-to-gas ratio. Smaller grains cluster on smaller scales; for $\tau_{\mathrm{s}}=0.01$ this corresponds to sub-GMC (core) scales, and for $\tau_{\mathrm{s}}=1$ this corresponds to super-GMC scales. If the disk is metal-poor, the dust-enhanced, high-density regions may be preferentially able to form low-mass stars.

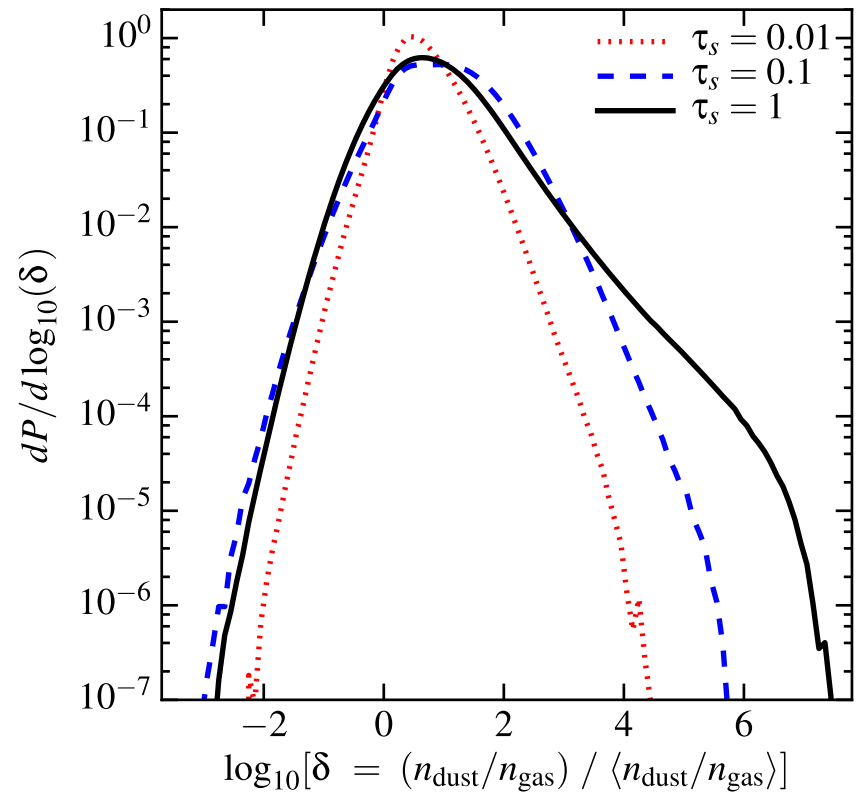

Figure 2. Predicted dust-to-gas ratio (relative to the mean) in the simulations in Figure 1. The results are time averaged in each case after the first few dynamical times when the simulations reach a statistical steady-state. In all cases, there are large fluctuations in the dust-to-gas ratio, as is evident in Figure 1 . The enhancements in the local dust density can, for large grains with $\tau_{\mathrm{s}} \gtrsim 0.01$, reach factors of $\sim 10^{4}$.

before enrichment by the first (Pop III) stars, these clouds form a broad spectrum of self-gravitating substructures, some of which eventually collapse to form stars (Barkana \& Loeb 2001; Greif et al. 2008; Wise et al. 2008; Wise \& Abel 2008; Pawlik et al. 2013). In our simulations, these randomly sample the dust-to-gas ratio fluctuations essentially independent of gas density. This is shown explicitly in Hopkins \& Lee 2016, where a series of scalefree simulations of pure aerodynamic dust dynamics are used to compare the variance in the dust-to-gas ratio distribution as a function of local density over $\sim 10$ decades in density. Hopkins (2013b) noted that any self-gravitating structure, by definition, must have a collapse time shorter than the eddy turnover time on the same scale, which is the coherence time of the dust-to-gas ratio fluctuations on that scale. Therefore, whatever amount of fluctuation appears in the dust-to-gas ratio on cloud scales $\sim R_{\text {cloud }}$, when a cloud crosses the critical density and collapses it is captured and conserved. Presumably, the captured grains will eventually shatter in collisions as their relative velocity increases while the core contracts; this will repopulate small grains and return mass to gas-phase metals, until they are eventually incorporated into the star (Hirashita 2010).

\subsubsection{Promoted Star Formation}

Various authors have argued that there is a critical minimum total metal and/or dust abundance $Z_{\text {crit }} \sim 10^{-5}-10^{-3} Z_{\odot}$ above which cooling is efficient, hence cold clouds can easily collapse and fragment down to stellar and substellar masses (which, unlike Pop III stars, ${ }^{6}$ could survive to the present day), and star formation is "normal" (see, e.g., Chiaki et al. 2014; Ji et al. 2014). ${ }^{7}$

Therefore, even if large positive enhancements in $Z_{d}$ are rare, they may be special under high-redshift conditions. Even if $\langle Z\rangle \ll Z_{\text {crit }}$, it is possible to form regions where, locally, $Z_{d} \gtrsim Z_{\text {crit. }}$. Because factor $\gg 100$ fluctuations are possible in $Z_{d}$, if $\gtrsim 10 \%$ of the metals are in large grains, this implies that galaxies with average metallicities as low as $\langle Z\rangle_{\min } \sim$ $0.01 Z_{\text {crit }} \sim 10^{-7}-10^{-5} Z_{\odot}$ might be able to produce regions with local $Z_{d}>Z_{\text {crit }}$ and form at least some low-mass stars. This extremely low $\langle Z\rangle_{\min }$ corresponds to halos with masses $M_{\text {halo }} \lesssim 10^{8.5}-10^{10} M_{\odot}$ (atomic cooling and smaller halos) enriched by at least one core-collapse event.

This actually makes it more likely that we might observe signatures of special $\mathrm{SNe}$ in metal abundance patterns in the long-lived low-mass stars of the next generation. Otherwise, without promoted star formation, enriching the gas in a $\sim 10^{10} M_{\odot}$ halo to a uniform $Z_{\text {crit }} \sim 10^{-3} Z_{\odot}$ would require

\footnotetext{
6 Assuming, of course, the conventional wisdom (but still unproven assumption) that Pop III stars are exclusively high-mass with short lifetimes.

7 Although others have argued that it may be possible to form at least some low-mass stars at any non-zero dust abundance, they agree that the probability of low-mass star formation increases rapidly with the dust abundance above $Z_{\text {crit }} \sim 10^{-5} Z_{\odot}$ (Dopcke et al. 2013).
} 
$\sim 10^{4} \mathrm{SNe}$, implying that the abundances of the most metalpoor stars should reflect IMF-averaged yields (consistent with our argument from the mass-metallicity relation in Section 1).

However, we might ask whether large grains alone are a sufficient coolant. The detailed calculations above have shown that dust cooling alone is indeed sufficient-in fact it is significantly more efficient than gas-phase metal coolingto produce fragmentation down to the substellar mass scale (the minimum estimated dust-phase metallicity for fragmentation $\sim 10^{-5} Z_{\odot}$, while for gas-phase metallicity it is $\sim 10^{-3} Z_{\odot}$ ). Schneider et al. (2006, 2012a, 2012b), Klessen et al. (2012), Nozawa et al. (2012), Dopcke et al. (2013), Meece et al. (2014), and others have shown that efficient fragmentation to subsolar masses requires the cooling time from dust $t_{\text {cool }}$ be comparable (or shorter than) to the dynamical (collapse) time $t_{\mathrm{dyn}} \sim 1 / \sqrt{G \rho}$ of a cloud by the time it reaches densities $n \gtrsim 10^{10}-10^{12} \mathrm{~cm}^{-3}$ (at much higher densities, the cloud becomes too optically thick to cool, regardless of the dust content; at much lower densities, turbulence can easily generate density fluctuations and create new gravitationally unstable regions, so the details of cooling are less important). This will drop the local Jeans mass to $\lesssim 0.1 M_{\odot}$. The gas cooling time (via large dust grains) is approximately $t_{\text {cool }} \sim 1$ / $\left(n_{\text {dust }} \pi a^{2} \delta v_{\text {dust-gas }}\right) \sim\left(\bar{\rho}_{\text {solid }} a\right) /\left(Z_{d} \rho_{\text {gas }} \delta v\right) \sim t_{\mathrm{s}} / Z_{d}, \quad$ so for sufficiently large $Z_{d}$, this is shorter than $t_{\mathrm{dyn}}$. However, most of the calculations of the critical dust abundance assume a normal grain size spectrum; if we depend only on large grains (for which the cooling is less efficient), the critical dust-phasemetallicity would naively be larger.

However, Nozawa et al. (2012) allow for arbitrary initial grain size distributions, and show that successful fragmentation does not depend on the initial grain size within the physical range we consider. Very crudely, we can illustrate this with the following argument: we can convert large grains to small (repopulating the size distribution) via collisions and shattering, and so increase the cooling rate (once bound in a contracting core, the large grains are expected to shatter or at least erode easily in collisions; Hirashita 2010). However this timescale is $\sim 1 /\left(n_{\text {dust }} \pi a^{2} \delta v_{\text {dust-dust }}\right)$, very similar to the direct cooling time-so it does not matter whether we directly cool down large grains or shatter/erode the grains first and cool down small grains/gas. Detailed calculations by Chiaki et al. (2014) and Chiaki \& Yoshida (2015) allowing for grain growth during collapse find consistent results.

In either case, then, if we assume that $\delta v$ tracks virial motions (or the sound speed, which should be similar in the limit where cooling is inefficient), then we obtain $t_{\text {cool }} \lesssim t_{\text {dyn }}$ when $Z_{d} \gtrsim 10^{-4} Z_{\odot} a_{\mu}\left(M_{\text {core }} / 100 M_{\odot}\right)^{-1 / 3}\left(n_{\text {gas }} / 10^{10} \mathrm{~cm}^{-3}\right)^{-2 / 3}$. So, near the mean densities of the galaxy, the large grains have no appreciable effect on cooling (but recall, turbulence dominates in this regime). But as the cloud contracts to higher densities, $t_{\text {cool }} / t_{\text {dyn }}$ decreases $\left(\propto n_{\text {gas }}^{-2 / 3}\right)$ until fragmentation occurs around the relevant densities $\left(n_{\text {gas }} \lesssim 10^{10} \mathrm{~cm}^{-3}\right.$ for $\left.Z_{d} \gtrsim 10^{-4} Z_{\odot} a_{\mu}\right)$. For detailed calculations, see the references above.

Indeed, there is growing observational evidence favoring the dust-cooling limit as the relevant limit on the stellar abundances of extremely metal-poor stars (Klessen et al. 2012; de Bennassuti et al. 2014; Ji et al. 2014). This suggests two things, that there is dust in extremely metal-poor environments and that dust is the critical physical enabler of low-mass star formation.
If star formation occurs as a consequence of dust-enhancement, it may in turn lead to a bias in the stellar abundances observed in the relic stars since only regions where $Z_{d} \gg\left\langle Z_{d}\right\rangle$ will form low-mass stars. We consider this below.

\subsubsection{Dust Grain Abundance Variations}

Dust-to-gas variations in cores translate to metal abundances. If a fraction $f_{\mathrm{p}}$ of a species $i$ is condensed in large grains, then a local fluctuation $\sim \delta=Z_{d} /\left\langle Z_{d}\right\rangle$ translates to a metal abundance $Z_{i}=\left(1-f_{\mathrm{p}}+\delta f_{\mathrm{p}}\right)\left\langle Z_{i}\right\rangle$. Because the grain density fluctuations are stochastic, a natural prediction of the scenario here is a variation in the metal abundances produced by concentrations of large grains.

What might we expect? Although the properties of grains (e.g., sizes and composition) vary significantly between regions of the Milky Way and nearby galaxies (see Draine 2003, for a review), there are some robust conclusions suggested by many observations. The large grains containing the most mass have sizes $\sim 0.1-100 \mu \mathrm{m}$; their cores appear to be a mix of nearly puregraphite (C) carbonaceous grains, and either olivine $\left(\mathrm{Mg}_{2} \mathrm{SiO}_{4}\right)$ or pyroxene $\left(\mathrm{MgSiO}_{3}\right)$ silicates (e.g., Kemper et al. 2004). ${ }^{8}$ Observed ice mantles are a mix of $\mathrm{H}_{2} \mathrm{O}$ (the dominant component), $\mathrm{CO}_{2}$, $\mathrm{CH}_{3} \mathrm{OH}, \mathrm{NH}_{3}, \mathrm{CH}_{4}, \mathrm{H}_{2} \mathrm{CO}, \mathrm{CO}$, and $\mathrm{XCN}$ (with $\mathrm{X}$ unknown), such that the average mass fraction is about $(0.65-0.73$, $0.12-0.15,0.04-0.12,0.08-0.1,<0.01)$ for $(\mathrm{O}, \mathrm{C}, \mathrm{N}, \mathrm{H}, \mathrm{X})$ (Gerakines et al. 1999; Chiar et al. 2000; Gibb et al. 2000; Keane et al. 2001; Draine 2003). In general, the ice-covered grains will always be the largest because the ice itself increases their size, and because ice mantles dramatically accelerate grain coagulation and sticking in grain collisions (Jones et al. 1994, 1996; Hirashita \& Yan 2009). The abundances of other species in large grains are, unfortunately, much less clear. $\mathrm{Na}$, for example, is depleted onto dust throughout the ISM, but it is not clear whether this is in large grains (Jenkins 2009). There can also be trace contributions from corundum $\left(\mathrm{Al}_{2} \mathrm{O}_{3}\right)$, nitrides $\left(\mathrm{Si}_{3} \mathrm{~N}_{4}\right)$, and silicon carbides $(\mathrm{SiC})$ at $\sim \mu \mathrm{m}$ sizes, as well as inclusions of $\mathrm{Ca}$ and $\mathrm{Ti}$ in silicates, but the relative abundance of these species (within grains) is usually low.

For illustrative purposes, we consider a highly simplified reference model following the default model fitted to cold clouds in Weingartner \& Draine (2001) and Draine (2003) in which the large grains are a specified mix of graphite and olivine, ${ }^{9}$ with ice mantles of $\mathrm{O}, \mathrm{C}, \mathrm{N}, \mathrm{H}$ with mass fractions of $0.66,0.13,0.12,0.09$. With this simplification, the effects of

\footnotetext{
8 Interestingly, observations indicate that the large silicates are iron-poor; so olivine $\mathrm{MgFeSiO}_{4}$ is either not present or more likely is a large component only in the small-grain population (Tielens et al. 1998; Sofia \& Meyer 2001; Molster et al. 2002a, 2002b).

9 Specifically, we follow Weingartner \& Draine (2001) and assume the large grain cores/mantles are either carbonaceous or silicate, where the carbonaceous grains are pure- $\mathrm{C}$ graphite chains and the silicate grains are amorphous olivine $\mathrm{Mg}_{2} \mathrm{SiO}_{4}$; they neglect inclusions as a small correction. The mass fraction in silicate versus carbonaceous grains as a function of size is given therein. Over the grain size range of interest, we assume that the ice mantles have uniform (mean) composition with an unknown mass fraction relative to the cores that we will treat as a free parameter (roughly, the ice density is $\approx 0.5$ times the core density, so for spherical grains, the mass fraction can be easily translated into a spatial "size" of the mantle. For ice, following the collection of observations synthesized in Draine (2003), we assume $\mathrm{H}_{2} \mathrm{O}$ is the dominant component, while $\left(\mathrm{CO}_{2}, \mathrm{CH}_{3} \mathrm{OH}, \mathrm{NH}_{3}, \mathrm{CH}_{4}, \mathrm{H}_{2} \mathrm{CO}, \mathrm{CO}, \mathrm{XCN}\right)$ have relative abundances

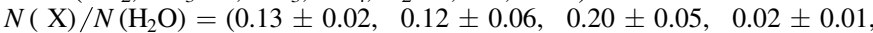
$0.04 \pm 0.02,0.06 \pm 0.03,0.03 \pm 0.01)$, which gives an approximate total mass fraction (summing over all species) of $(0.66,0.12,0.12,0.09,0.01)$ for $(\mathrm{O}, \mathrm{C}, \mathrm{N}, \mathrm{H}, \mathrm{X})$ ("X" here represents all other species; we take it to uniformly sample a solar-abundance-ratio distribution, but its abundance is so small as to be negligible).
} 
dust on stellar metal abundances are specified by two parameters: the local dust-to-gas ratio and the size of the ice mantles.

Of course, the mean grain composition (let alone the distribution of grain compositions) even at present day is uncertain; our "default" model here is intended only to be illustrative for one specific example (see below). In most of what follows, we prefer to show a range of abundance ratios corresponding to the observed range in ISM dust. Even in the dominant species $(\mathrm{CNO}, \mathrm{Mg}, \mathrm{Si})$ there can be variations of $\sim 0.3$ dex or larger in abundance ratios. Moreover, at high redshift there have been suggestions that the dust composition may differ, e.g., becoming mostly silicate based (as opposed to carbon based; see Cherchneff \& Dwek 2010, and references therein). Obviously, in the absence of ice mantles, this would imply little or no $\mathrm{C}$ in dust, hence this model would predict only enrichment in $\mathrm{Mg}, \mathrm{Si}$, and $\mathrm{O}$; however, in the presence of significant ice mantles, most of the predicted carbonaceous mass in the grains is actually in the ice mantle, and a slightly larger mantle could easily compensate for the absence of carbonaceous/graphite cores in depleting the gas-phase carbon onto grains.

\section{Observational Signatures}

\subsection{Single-star Abundance Patterns}

Figure 3 considers the stellar abundances of a prototypical carbon-enhanced metal-poor star, CS29498-043 $([\mathrm{Fe} / \mathrm{H}]=$ -3.52) from Tominaga et al. (2014). ${ }^{10} \mathrm{We}$ compare this to the IMF-averaged yields of core-collapse $\mathrm{SNe}$ with metal-free progenitors from Heger \& Woosley (2010). Although it is perfectly plausible that a small number of SNe are responsible for the enrichment at these low metallicities, we plot IMFaveraged values to give an idea of the mean abundance patterns expected and/or those of the low metallicities owing to even a modest number of events mixed together and (potentially) diluted. Other models, such as Woosley \& Weaver (1995) or Nomoto et al. (2006), give similar results even if we freely vary the progenitor metallicity (best-fit $Z \sim 0.001$ ) or hypernovae fraction (in the latter). Overall, the IMF-averaged models do well for elements heavier than $\mathrm{Si}$, and for $\mathrm{Na}$ and $\mathrm{Al}{ }^{11}$ However, there is a serious discrepancy (a factor of 20-300) with the light elements ( $\mathrm{CNO}, \mathrm{Mg}, \mathrm{Si}$ ); the models are not qualitatively correct for these elements as they predict subsolar $\mathrm{C}, \mathrm{N}, \mathrm{Mg}$. Adding $\mathrm{SNe}$ Ia components, changing progenitor metallicities, or removing the hypernovae component from Nomoto et al. (2006) only increases these discrepancies.

Of course, it is possible to find $\mathrm{SNe}$ nucleosynthesis models that better fit the stellar abundances by allowing for single or arbitrary mixes of progenitor stars/explosions and then, for example, adjusting the progenitor abundances (although this begs the question), explosion energies, masses, density profiles, rotational support, and mixing efficiencies, and by invoking prior failed explosion/fallback episodes or jet ejection of certain species. A simple search over progenitor masses,

\footnotetext{
${ }^{10}$ The observations are compiled from many sources in Tominaga et al. (2014); we plot the uncertainty-weighted average of the values there whenever multiple literature values exist.

11 There are some well-known modest discrepancies with the underproduction of $\mathrm{Ti}$ and overproduction of $\mathrm{Mn}$; they persist even in solar-metallicity populations and are probably due to NLTE effects and other detailed modeling discrepancies.
}

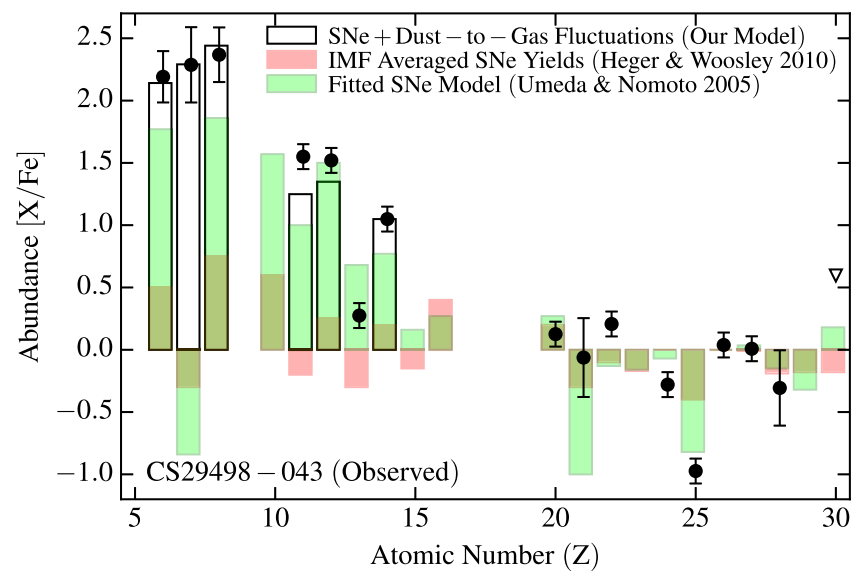

Figure 3. Stellar abundance pattern of a typical carbon-enhanced metal-poor star $(\mathrm{CS} 29498-043 ;[\mathrm{Fe} / \mathrm{H}]=-3.52$; black points show data from Tominaga et al. 2014; arrows indicate upper limits). The IMF-averaged yields of corecollapse SNe with metal-free progenitors from Heger \& Woosley (2010) are shown by red histograms. Adding hypernovae, freeing the progenitor metallicity, or using the IMF-averaged yields from Nomoto et al. (2006) does not improve the agreement; clearly "mean" yields fail to explain this star. The blue histogram shows the best-fit abundance pattern from a single-explosion progenitor model in Umeda \& Nomoto (2005), searching over the progenitor metallicity, mass, and explosion energy, and freely varying mixing and fallback parameters; this provides a plausible fit. However, black histograms show the prediction if we take the Heger \& Woosley (2010) IMF-averaged yields and additionally allow for local variations in the dust-to-gas ratio according to the simulations. We take our default dust model from the text, and allow the dustto-gas ratio and ice mantle size (two parameters) to freely vary within the range simulated, and show the best-fit result. The unusual abundances of light elements $\mathrm{C}, \mathrm{N}, \mathrm{O}, \mathrm{Mg}, \mathrm{Na}$, and $\mathrm{Si}$ could all, in principle, be explained by a local dust overabundance in the region that was able to successfully form stars (under these metal-poor conditions), as opposed to unusual progenitor $\mathrm{SNe}$

metallicities, mixing parameters, and explosion energies in Umeda \& Nomoto (2005) gives the best-fit single-progenitor explosion model shown in Figure 3, based on a lowenergy $\mathrm{SNe}$ with fallback and mixing $\left(25 M_{\odot}, Z=0\right.$ progenitor with explosion $E=10^{51} \mathrm{erg}$ ). Adding the additional degrees of freedom noted above, the best-fit model in Tominaga et al. (2014) still predicts discrepancies in N, Na, Al, and Si of -1.8 , $-0.4,+0.9$, and $+0.25 \mathrm{dex}$, respectively, so it does not represent a substantial improvement. In either case, this is of course a much better fit to the data-and indeed represents one plausible channel for the formation of the star.

However, if we simply add our reference dust model to the IMF-averaged yields, allowing the dust-to-gas ratio $\left(Z_{d} /\langle Z\rangle\right)$ and size of ice mantles to be two unknown parameters, we can almost perfectly reproduce the (five) discrepant light elements. ${ }^{12}$ From the best-fit, the implied enhancement in the dust abundance in the star-forming region is $Z_{d} \sim 50-100\left\langle Z_{d}\right\rangle$ (well within the predicted range of our simulations). If we assume that the ices surround carbonaceous grains, the best-fit abundances imply mantles of sizes that are $\sim 2-3$ times the core sizes (i.e., $\sim 10 \%$ of $\mathrm{C}$ in cores, the rest in ice, implying physical mantle sizes up to $\sim 1 \mu \mathrm{m}$ ). If we assume that the ices surround silicates, the mantle sizes are $\sim 3$ times the core sizes, and $\sim 5 \%$ of the $\mathrm{Si}$ mass is in cores. These inferences are all consistent with common observed properties in cold, neutral

\footnotetext{
12 Note that if we assume the $\mathrm{Na}$ is preferentially depleted onto large grains (which is by no means clear), we can also reproduce the observed $\mathrm{Na}$ abundance with dust; however, this could just as well be accounted for by metal-poor core-collapse $\mathrm{SNe}$
} 
regions of the local ISM (Witt et al. 2001; Weingartner \& Draine 2001; Costantini et al. 2005; Jenkins 2009). ${ }^{13}$

\subsection{Shape of the Stellar and Dust Abundance Distributions}

Figure 4 plots the stellar abundances (as in Figure 3) for each star in the Tominaga et al. (2014) compilation. The scatter is much larger in the light elements. The scatter in $\mathrm{C}$ and the icerelated species, possibly due to abundance variations caused by the largest grains, is $\sim 1$ dex. This is comparable to a nearly constant $\sim 0.2$ dex spread in all the elements from $\mathrm{Ca}$ to $\mathrm{Zn}$ where we expect weaker dust effects.

There are multiple possible explanations for this. However, if turbulence drives dust-density fluctuations, an approximately lognormal distribution of dust-density enhancements $\delta$ is predicted; this translates to a similar distribution in the largegrain species, but with a cutoff at low metal abundances (because some order-unity fraction of the metals is not in large grains). So in Figure 4, we collect several compilations of metal-poor stars from the literature: the Tominaga et al. (2014) sample above, as well as the Frebel (2010), Aoki et al. (2013), and Yong et al. (2013) samples. For each, we plot the cumulative distribution of enhancements in the large-grain elements. These are highly incomplete, inhomogeneous samples, so the enhancement distribution should be regarded with great caution, but they represent nearly all the metal-poor populations known with reliable stellar abundance determinations.

We compare this to a very simple toy model for promoted star formation based on our simulations. For each simulation, we construct $10^{6}$ random realizations. We draw a galaxyaverage $[\mathrm{Fe} / \mathrm{H}]$ to match the distribution of stars in the Tominaga et al. (2014) sample; apply our reference model (for IMF-averaged yields and dust), assuming that $f_{\mathrm{p}} \sim 1 / 2$ of the $\mathrm{CNO}$ elements are in the large grains (in ices or cores); calculate the total CNO metallicity (gas+dust) within each resolution element (above the mean density); and randomly decide whether each should form a star. Lacking any physical model for promoted star formation we simply assume that the probability of star formation is proportional to the total metallicity at these low metallicities (which is arbitrary, but our intention is simply to represent the qualitative behavior we expect). Given the extreme simplicity of this model, it is remarkable how well the predictions for $\tau_{\mathrm{s}} \sim 0.1-1$ appear to match the observed distributions.

\subsection{CNO Elements, Carbonaceous Grains, and Ices}

In Figure 5, we consider the ratio of the $\mathrm{CNO}$ species observed. We caution that the ratios of the individual species involved here are subject to serious stellar evolutionary effects after the stars form, but even given this caveat, we wish to highlight that many stars have stellar abundance ratios similar to those found in ISM ices. For ice with the mean observed mixture of components in our reference model, we expect [N/ $\mathrm{O}]$ between -0.5 and +0.2 , and $[\mathrm{C} / \mathrm{O}]>-0.5$ depending on

\footnotetext{
${ }^{13}$ For example, the mass ratio of mantles to cores can easily be obtained if initial grain cores are produced with relatively small sizes $\sim 0.01 \mu \mathrm{m}$ (as might be expected for SNe-produced grains after a reverse shock; see Bianchi \& Schneider 2007; Nozawa et al. 2007), after which CNO is quickly depleted into ice mantles on the grains (which simply suggests that similar-sized mantles would be produced on all grains with sizes $\sim 0.02-0.03 \mu \mathrm{m}$; Jones et al. 1996). Being ice-coated, the grains would then efficiently grow by sticking/ coagulation (Jones et al. 1994).
}
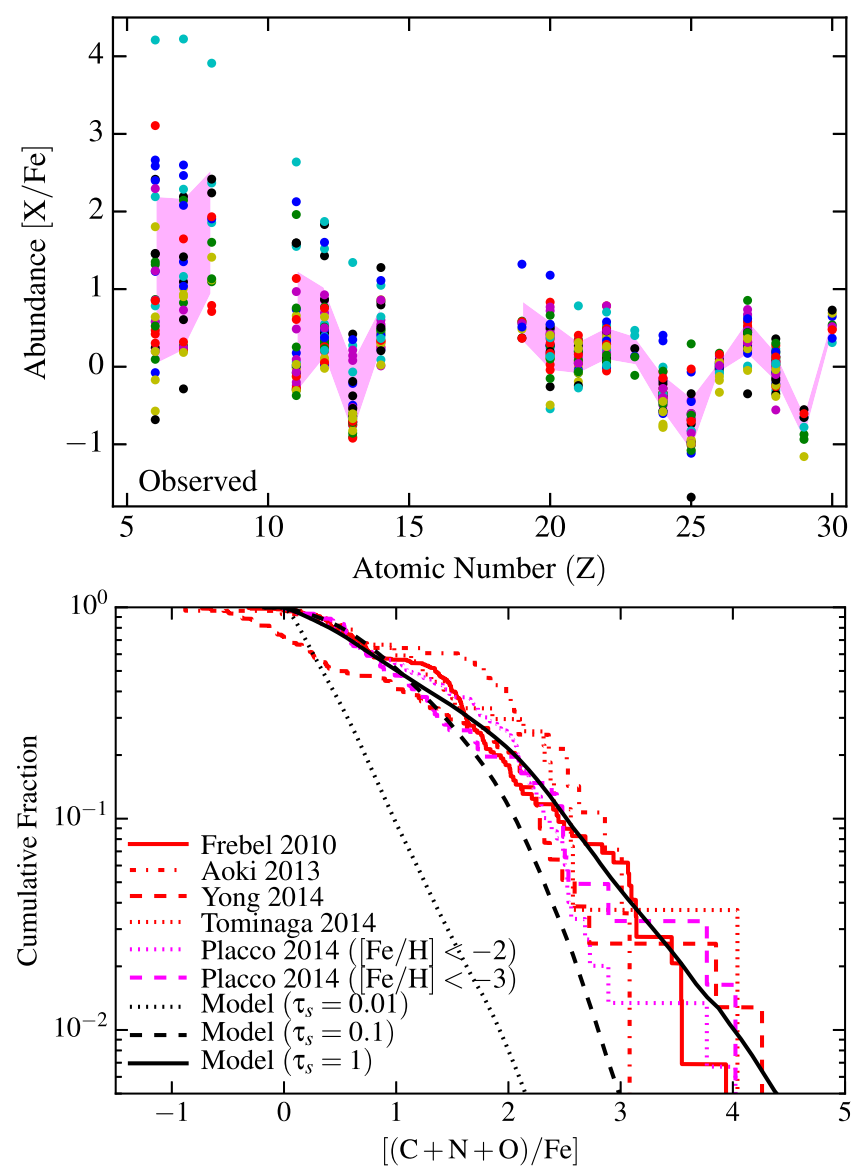

Figure 4. Top: stellar abundances of all stars in the Tominaga et al. (2014) sample; the shaded region shows the $1 \sigma$ scatter. As is well known, the light element abundances (those which we compared in Figure 3) vary more dramatically than the heavy element abundances. Bottom: distribution of $[\mathrm{C} / \mathrm{Fe}]$ from the observational compilations in Frebel (2010), Aoki et al. (2013), Yong et al. (2013), Tominaga et al. (2014). We compare the predicted distribution from simulations with our default dust model, assuming that a fraction of $\sim 0.5$ of the $\mathrm{C}$ is in large dust grains (either cores or mantles). Although there are many physical mechanisms that can lead to $[\mathrm{C} / \mathrm{Fe}]$ variations, and the observational samples are incomplete, the agreement between observations and simulations is suggestive.

the ratio of ice mantle to underlying carbonaceous grain mass. If we consider a simple Pearson test, the enhancements $[\mathrm{C} / \mathrm{Fe}]$, $[\mathrm{N} / \mathrm{Fe}]$, and $[\mathrm{O} / \mathrm{Fe}]$ are all correlated with each other ( $p_{\text {uncorrelated }} \ll 10^{-4}$ in both the Tominaga et al. 2014 and Frebel 2010 samples), albeit with significant scatter, as expected in the models here (as opposed to anti-correlated if stellar evolution were dominant).

\subsection{Distinguishing Between SNe Yields and ISM Dust with Mg, $\mathrm{Si}$, and $\mathrm{Ca}$}

A more robust constraint can come from the behavior of species in massive silicates. First, we should stress that because of expected variation in the ice mantle covering and size distributions of silicate and carbonaceous grains (and the fact that grains with different $\tau_{\mathrm{s}}$ will, even in the same location in the same galaxy, be affected differently), we do not necessarily predict a strong correlation between $\mathrm{C}$ and $\mathrm{Si}$ enhancements. However, there should be a positive trend where stars with enhanced $\mathrm{C}$ are at least more likely to have enhanced variation in $\mathrm{Si}$; indeed, a Pearson test confirms this $\left(p_{\text {uncorrelated }} \sim 10^{-5}\right)$, 


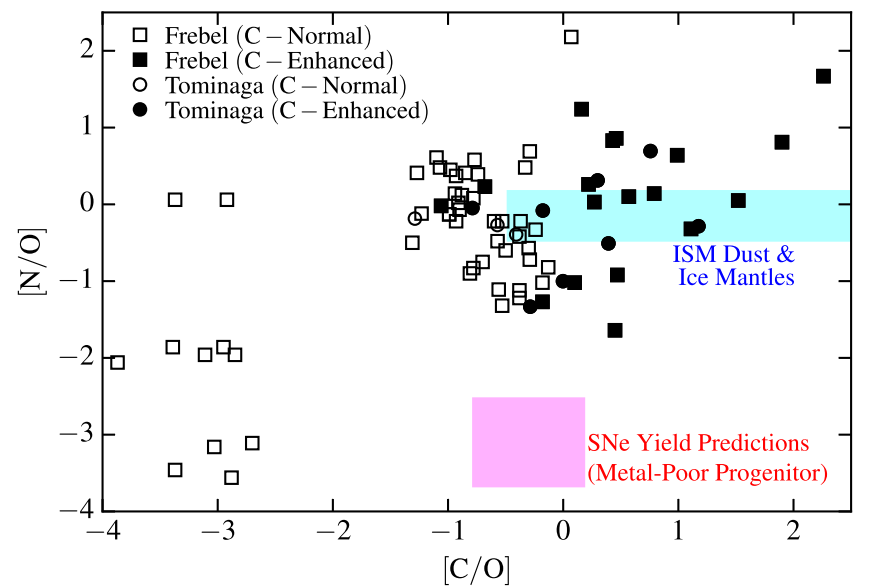

Figure 5. Distribution of CNO elements in observed metal-poor stars, from the same compilations as Figure 4. Depending on the ratio of ice mantle to core size, large grains in the simple Draine (2003) dust model reside anywhere within the blue shaded area. The red shaded area represents the range of yields for standard $\mathrm{SNe}$ of any progenitor mass and explosion energy with low metallicities $(Z<0.001)$ in the Woosley \& Weaver (1995) models (the Nomoto et al. (2006) models are similar). The CNO abundances are highly degenerate with stellar evolution and pollution by winds, but the similarity of the observed abundances in the C-enhanced stars to ices, and disagreement with the low $\mathrm{N}$ enrichment predicted by metal-poor SNe models, is suggestive of star formation in dust-enhanced regions.

and it is plain "by eye" in both the Tominaga et al. (2014) and Frebel (2010) samples.

However, where $\mathrm{Si}$ is enhanced (because of dust) our reference model more robustly predicts a correlated enhancement in $\mathrm{Mg}$ since it comes from the same grains. Indeed, within the metal-poor population we find that $([\mathrm{Fe} / \mathrm{H}]<-2.5),[\mathrm{Si} / \mathrm{Fe}]$, and $[\mathrm{Mg} / \mathrm{Fe}]$ are more significantly correlated with each other $\left(p_{\text {uncorrelated }} \sim 10^{-8}\right)$ than with the heavier species $\mathrm{K}, \mathrm{Ca}, \mathrm{Sc}, \mathrm{Ti}, \mathrm{V}, \mathrm{Cr}, \mathrm{Mn}, \mathrm{Co}, \mathrm{Ni}, \mathrm{Cu}, \mathrm{Zn}$, $\left(p_{\text {uncorrelated }} \sim 0.1-1\right) .{ }^{14}$ Figure 6 shows this more quantitatively. We plot $[\mathrm{Mg} / \mathrm{Si}]$ and $[\mathrm{Mg} / \mathrm{Ca}]$ versus $[\mathrm{C} / \mathrm{Fe}]$. If the silicate grain cores are a mix of olivine $([\mathrm{Mg} / \mathrm{Si}] \sim+0.3)$ and pyroxene $([\mathrm{Mg} / \mathrm{Si}] \sim-0.05)$, we expect $[\mathrm{Mg} / \mathrm{Si}]$ in a relatively narrow range. Figure 6 confirms this, especially for stars with $[\mathrm{C} / \mathrm{Fe}] \gtrsim 0.5$ (given measurement errors, $>90 \%$ of the stars with this $[\mathrm{C} / \mathrm{Fe}]$ are consistent with the predicted range in $[\mathrm{Mg} / \mathrm{Si}]){ }^{15}$

For comparison, in burning models the production of $\mathrm{Mg}$ and $\mathrm{Ca}$ (produced by similar processes in stars of similar mass) should be much more closely linked than $\mathrm{Mg}$ and $\mathrm{Si}$. For pure, metal-poor, IMF-integrated ejecta, these models predict $[\mathrm{Mg} / \mathrm{Ca}] \sim-0.3$ with relatively small scatter (Woosley \& Weaver 1995). In fact, we find that nearly every star is enhanced above this level, as predicted if the star-forming region is dust enhanced. Interestingly, if we restrict the model to more metal-rich ejecta that are not light-element enhanced $([\mathrm{Fe} / \mathrm{H}]>-2.5$, with $[\mathrm{Mg} / \mathrm{Fe}]<0.5)$, then the correlation between $\mathrm{Mg}$ and $\mathrm{Ca}$ is good. Within that population, we expect the fraction of dust-enhanced stars to be small.

\footnotetext{
${ }^{14}$ There are marginally significant correlations between $[\mathrm{Si} / \mathrm{Fe}]$ or $[\mathrm{Mg} / \mathrm{Fe}]$ and $[\mathrm{Na} / \mathrm{Fe}]$ or $[\mathrm{Al} / \mathrm{Fe}]$, which could stem either from enrichment by prior $\mathrm{SNe}$ or dust contamination (with some amount of dust containing $\mathrm{Na}$ and $\mathrm{Al}$, as discussed above).

15 There also appear to be some stars, albeit few, that have highly enhanced $\mathrm{Ca}$ at low metallicities (see Lai et al. 2009).
}
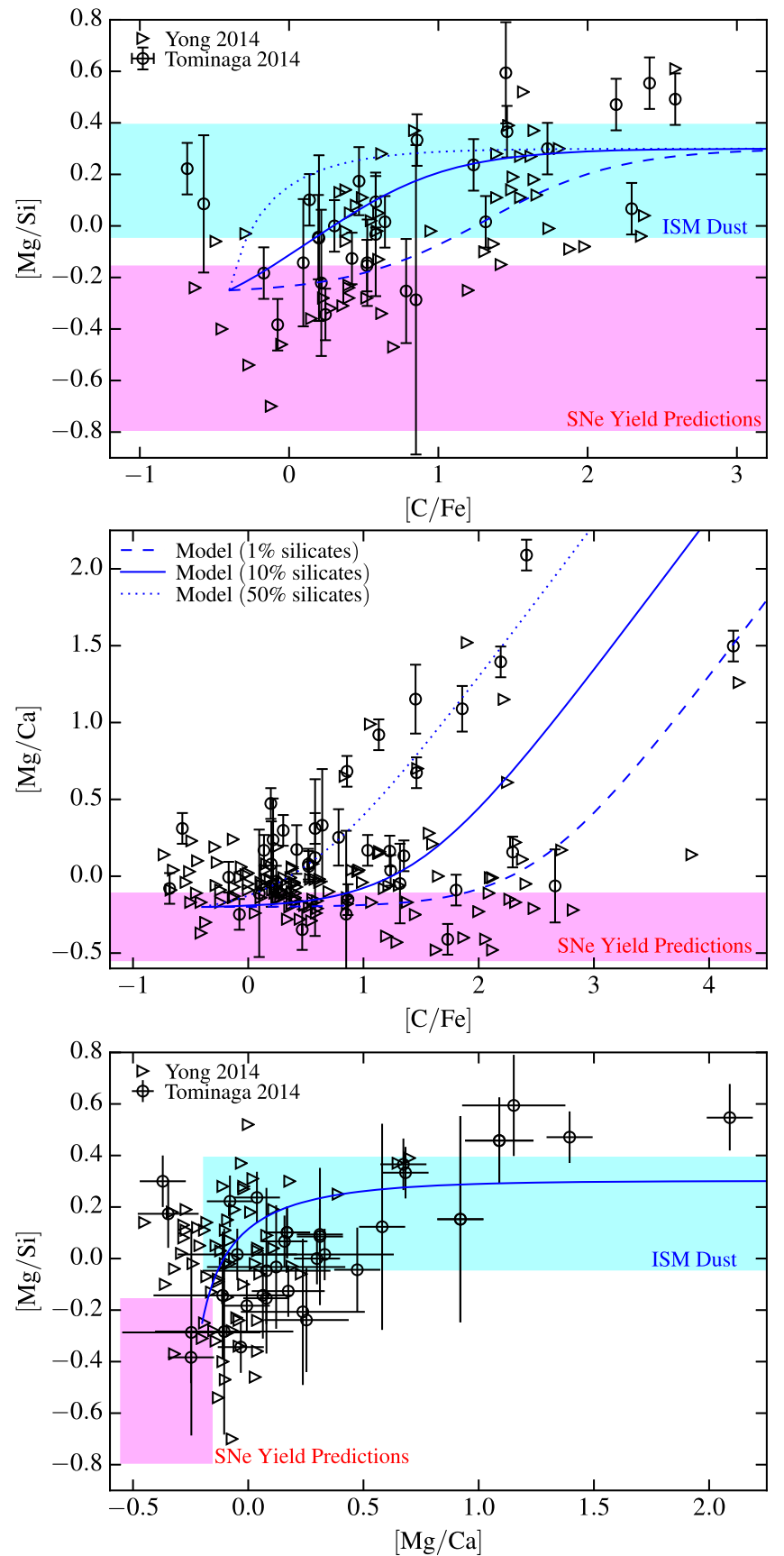

Figure 6. Top: observed $[\mathrm{Mg} / \mathrm{Si}]$ vs. $[\mathrm{C} / \mathrm{Fe}]$ in metal-poor stars, as in Figure 5. Middle: observed $[\mathrm{Mg} / \mathrm{Ca}]$ vs. $[\mathrm{C} / \mathrm{Fe}]$ in the same stars. Bottom: $[\mathrm{Mg} / \mathrm{Si}]$ vs. $[\mathrm{Mg} / \mathrm{Ca}]$. As in Figure 5, the range of $[\mathrm{Mg} / \mathrm{Si}]$ in typical observed ISM silicate grains (at low redshift) is shown in the shaded blue range; the range of yields predicted for different individual progenitor masses and energies in metal-poor $\mathrm{SNe}$ is shown in shaded red. SNe models (including hypernovae and metal-rich $\mathrm{SNe}$; see Nomoto et al. 2006) tend to predict a narrow range of $[\mathrm{Mg} / \mathrm{Ca}]$. But the C-enhanced stars also tend to be enhanced in $[\mathrm{Mg} / \mathrm{Ca}]$ and have similar $[\mathrm{Mg} / \mathrm{Si}]$ to the observed silicate grains. This is predicted if they form in dustenhanced regions. Assuming our reference model for grains in a background with IMF-averaged yields, we predict that dust-enhanced stars lie along the tracks shown as blue lines, where the track depends on the (unknown) ratio of the total mass in silicate grains to carbonaceous grains plus ices at the grain sizes that are experiencing density fluctuations. In the bottom panel, the model tracks lie on top of one another.

\section{Discussion and Conclusions}

Provided dust grains with sizes $\gtrsim 0.01-0.1 \mu$ m exist in highredshift, predominantly neutral galaxies, we show that the 
relatively weak coupling between the dust and gas means that these grains can experience orders-of-magnitude fluctuations in their local number density (dust-to-gas ratio).

This means there can be efficient cooling and low-mass (Pop-II) star formation in clouds with high local dust density, even when the galaxy-average metallicity is extremely low $(\langle\log [Z / H]\rangle \ll-5)$. In other words, second-generation star formation can begin (albeit stochastically) almost immediately after the first dust is produced (via Pop-III winds or $\mathrm{SNe}$ ) without waiting for any galaxy-wide enrichment threshold to be reached. We refer to this mechanism as promoted star formation.

This may also have significant impact on the stellar abundance patterns of certain elements in the stars that form from these clouds. We show that this can naturally explain otherwise unusual abundance patterns in metal-poor stars, including the large carbon-enhanced (and CNO-enhanced) population, and stars with elevated, tightly correlated $\mathrm{Si}$ and $\mathrm{Mg}$ without $\mathrm{Ca}$ enhancement. This would explain growing observational indications of independent formation channels for the observed CEMP-no and carbon-normal metal-poor stars (Norris et al. 2013). Compared to SNe nucleosynthesis models, better fits to the stellar abundances are found with fewer free parameters for a substantial subpopulation of stars using a simple standard model of dust chemistry coupled to direct numerical simulations of dust dynamics. This simple dustenhancement model also naturally predicts a quasi-lognormal distribution of abundances, in good agreement with observations, and typical abundance ratios of $\mathrm{CNO}$ and $\mathrm{Si}, \mathrm{Mg}$, and $\mathrm{Ca}$, which are observed but are difficult if not impossible to explain in most $\mathrm{SNe}$ nucleosynthesis models.

The key theoretical assumption here is that there is dust that contains a non-negligible fraction of metals in such lowmetallicity galaxies. According to some models, this is unlikely. However, a growing body of observations (as well as newer theoretical models; see, e.g., Hirashita et al. 2014; Mattsson et al. 2014; Marassi et al. 2015, 2014) indicates it may be inevitable. Absorption in gamma-ray bursts, quasars, and lensed galaxies at high redshifts (many at $z \gtrsim 5-7$ ) indicates they have normal dust-to-metal ratios, despite metallicities as low as $[Z / H] \sim-2.5$ (Cucchiara et al. 2011; Chen et al. 2013; De Cia et al. 2013; Kuo et al. 2013; Zafar \& Watson 2013; Dwek et al. 2014; Sparre et al. 2014). In fact, observational compilations from $z \sim 0-10$ suggest there is little significant evolution in the dust-to-metal ratio (references above), extinction curve (Cucchiara et al. 2011; Sparre et al. 2014), maximum dust sizes (grains $>0.1 \mu \mathrm{m}$ required at all times; Updike et al. 2011; Hirashita 2012; Kuo et al. 2013), or the carbon/silicate ratio in the large grains (though this is more uncertain; see, e.g., Kuo et al. 2013; Dwek et al. 2014). ${ }^{16}$ It has also been observed that $\mathrm{SNe}$ can directly produce large quantities of dust (an order unity fraction of the ejecta metals) with especially large grain sizes up to $\sim 10 \mu \mathrm{m}$ that would survive reverse shocks-these appear especially in

\footnotetext{
${ }^{16}$ It has been noted by many authors that in high-redshift galaxies and local low-metallicity galaxies like the LMC and SMC, the relative weakness of the $2175 \AA$ feature suggests a lower ratio of graphite dust to silicate dust (see, e.g., Pei 1992; Richards et al. 2003; Hopkins et al. 2004; Maiolino et al. 2004; Li et al. 2008; Perley et al. 2010, 2011; Updike et al. 2011; Schady et al. 2012; Zafar et al. 2012). However, this only constrains carbonaceous grains with sizes $\lesssim 10 \mathrm{~nm}\left(a_{\mu}<0.01\right)$, which is irrelevant for our purposes; in fact in most models, if these grains are removed, carbon is incorporated in larger grains, enhancing the effects we propose here.
}

$\mathrm{SNe}$ of types that some authors have speculated occur preferentially at low metallicity (e.g., IIn; De Marchi \& Panagia 2014; Gall et al. 2014; Kochanek 2014; Marassi et al. 2015). The same may be true of early stellar winds (Nozawa et al. 2014). Finally, more and more observational data favor dust as the critical coolant enabling fragmentation and formation of the observed, low-mass extremely metal-poor stars (Klessen et al. 2012; Schneider et al. 2012a; Chiaki et al. 2014; de Bennassuti et al. 2014; Ji et al. 2014); this suggests that dust must have been present in substantial amounts relative to gas when these stars formed.

Given the presence of dust in a neutral, modestly MHDturbulent gas disk with a ratio of dust stopping time to orbital time $\tau_{\mathrm{s}} \sim 1$, there are many analogies between this problem and the well-studied problem of dust dynamics in protostellar disks. The most important factors that differentiate the galactic case is (1) the absolute scales are different, so micron-sized dust in a galactic mini-halo behaves like meter-sized boulders in a protoplanetary disk; (2) the gas is compressible, which can enhance fluctuations in the dust-to-gas ratio; and (3) selfgravity is much more important in the galactic case, while the mean metallicities are much lower, so we do not expect to form super-planetesimals, but rather to promote normal star formation via cooling in regions where the dust abundance is relatively high.

What makes high-redshift galaxies special compared to lowredshift galaxies is (1) they are primarily neutral, even at surface densities where $\tau_{\mathrm{s}} \sim 1$. In the Milky Way, these densities would be fully ionized, so Coulomb and Lorentz forces would dominate the dust dynamics; moreover, the much stronger radiation field in the Milky Way suppresses ice formation and grain coagulation. And (2) the early galaxies are metal-poor, so cooling and star formation are expected to be less efficient under mean conditions. As such, regions with unusual dust/metal abundances might be the only regions capable of low-mass star formation, and therefore the observed stellar relics of this era will be preferentially biased toward these abundances. In contrast, while we do predict that qualitatively similar processes can occur in at least some neutral regions of galaxies today (certain large molecular clouds, for example), the dust-enhanced stars would represent only a miniscule fraction of the stellar populations forming in these regions (see Hopkins 2014).

This raises many questions that merit further study. The same instabilities shown here may dramatically enhance grain formation and growth in protogalaxies; with nominal clumping factors $\left\langle\rho_{\text {dust }}^{2}\right\rangle /\left\langle\rho_{\text {dust }}\right\rangle^{2}$ reaching $\sim 1000$, these effects need to be incorporated into dust growth models. Our dust dynamics simulations are also being extended to global disk simulations, with explicit models for Lorentz forces (neglected here). But even these are not actual star formation simulations. However, the simple equations of dust dynamics could be incorporated into self-consistent simulations of star formation which include detailed chemistry, dust+gas cooling, self-gravity, and dynamical enrichment models. This would enable more detailed, quantitative predictions for the importance of promoted star formation in dust-enhanced regions.

Perhaps most importantly, this motivates more detailed study and models of dust chemistry in high-redshift galaxies. For the sake of simplicity (and to make progress) we adopted a dust model calibrated to local observations, but this is almost certainly incorrect (although it provides a surprisingly good fit 
to observations). We believe that this is the largest uncertainty in predicting observable stellar abundance signatures from dust. Of course the elements heavier than $\mathrm{H}$ and $\mathrm{He}$ in dust must originally come from nucleosynthesis; therefore, if different gas-phase metal abundance ratios are present at high redshift, one might expect that the dust will reflect this. In the limit where the large dust grains perfectly trace the gas-phase metal abundances, it obviously becomes impossible to identify the mechanism we propose here via stellar abundance patterns (although this limit is unlikely, given the range in condensation temperatures of different species). However, such mechanisms could still be critical in producing dust-to-gas variations that allow "promoted" star formation.

We thank Jessie Christiansen, Evan Kirby, and Selma de Mink for many helpful discussions during the development of this work. Support for P.F.H. was provided by an Alfred P. Sloan Research Fellowship, NASA ATP Grant NNX14AH35G, and NSF Collaborative Research Grant \#1411920 and CAREER grant \#1455342. Numerical calculations were run on the Caltech compute cluster "Zwicky" (NSF MRI award \#PHY-0960291) and allocation TG-AST130039 granted by the Extreme Science and Engineering Discovery Environment (XSEDE) supported by the NSF. C.C. is supported by the Packard Foundation, NASA grant NNX13AI46G, and NSF grant AST-1313280.

\section{Appendix \\ Details of the Simulations}

\section{A.1. Numerical Method}

The simulations in the text are from the set in P. F. Hopkins \& H. Lee (2015, in preparation). They solve the standard equations of magnetohydrodynamics (MHD) using GIZMO (Hopkins 2015), a mesh-free, Lagrangian finite-volume Godunov code that takes advantage of both grid-based and smoothed-particle hydrodynamics (SPH) methods. In Hopkins (2015) and Hopkins \& Raives (2016), we consider extensive tests of the method, and show that GIZMO agrees very well with state-of-the-art moving-mesh and grid-based adaptivemesh refinement codes on both sub- and supersonic MHD turbulence.

The turbulent driving routines are the same as those used for these tests (see Hopkins 2013a, 2015 for details), and follow Bauer \& Springel (2012). The box is stirred via the same method as Schmidt et al. (2008), Federrath et al. (2008), and Price \& Federrath (2010): a narrow range of modes is driven in Fourier space as a purely solenoidal Ornstein-Uhlenbeck process. After a short period of initial adjustment $(\sim 1$ turbulent crossing time), this maintains a quasi-steady-state Mach number and turbulent cascade; the statistical properties of the simulations remain constant after this time.

We adopt the well-known shearing-sheet approximation (for details see, e.g., Guan \& Gammie 2008). Here we solve the vertically integrated equations for dust and gas in 2D (following radial/azimuthal $R, \phi$ coordinates) in a local frame that co-rotates with circular orbits with a frame-centered orbital frequency $\Omega$. This amounts to adopting standard shear-periodic boundary conditions, with the centrifugal and Coriolis accelerations $\boldsymbol{a}=2 q x \Omega^{2} \hat{x}+2 \boldsymbol{v} \times(\Omega \hat{z})($ where $q \equiv-d \ln \Omega / d \ln R)$.

Following most previous studies (see, e.g., Hogan et al. 1999; Johansen \& Youdin 2007; Carballido et al. 2008; Johansen et al. 2009; Bai \& Stone 2010b; Pan et al. 2011), we represent the dust via "super-particles," each of which represents an ensemble of grains with trajectories integrated on the fly. Following Draine \& Salpeter (1979), the grains obey the equations of gravity along with the drag equation

$$
\begin{gathered}
\frac{d \boldsymbol{u}_{d}}{d t}=-\frac{\boldsymbol{u}_{d}-\boldsymbol{u}_{\mathrm{gas}}}{t_{s}} \\
t_{s} \equiv \frac{\pi^{1 / 2}}{2 \sqrt{2}}\left(\frac{\bar{\rho}_{\mathrm{solid}} a}{c_{s} \rho_{\mathrm{gas}}}\right)\left(1+\left|\frac{3 \pi^{1 / 2}}{8} \frac{\boldsymbol{u}_{d}-\boldsymbol{u}_{\mathrm{gas}}}{c_{s}}\right|^{2}\right)^{-1 / 2},
\end{gathered}
$$

where $\boldsymbol{u}_{d}$ is the grain velocity, $d / d t$ is a Lagrangian derivative, $c_{s}$ and $\rho_{\text {gas }}$ are the isothermal sound speed and density of the gas, $\bar{\rho}_{\text {solid }}$ is the internal (material) grain density, and $a$ is the grain radius. The time-integration scheme is described in P. F. Hopkins \& H. Lee (2015, in preparation). Note that this is the proper expression for supersonic flows, and both $c_{s}$ and $\rho_{\text {gas }}$ are the values evaluated at the grain position, which can be spatially variable. This is the major difference between our study and previous works on protoplanetary disks, which assume Mach numbers $\mathcal{M} \ll 1$, and therefore drop the velocity-dependent term in $t_{s}$ and take $\rho_{\text {gas }}=$ constant.

\section{A.2. Approximations}

As noted in the text, this assumes that grains are in the Stokes regime which is trivially satisfied for $a \ll 10^{13} \mathrm{~cm}$. Because the absolute metallicities $Z_{d}$ of interest are low $\left([Z / H] \lesssim-2.5\right.$, hence $\left.\left\langle Z_{d}\right\rangle \lesssim 10^{-5}\right)$, we can safely neglect back-reaction (i.e., the momentum loss from gas onto grains), which is only important for local $Z_{d} \gg 1$. Also, as noted in the text, radiation pressure and Coulomb forces are negligible in the regime we simulate (primarily neutral gas, not in the $\mathrm{H}$ II region of individual stars).

The case of Lorentz forces on dust is less clear. Adopting equipartition magnetic fields and a mean grain charge as a function of grain size estimated in Draine \& Sutin (1987), and assuming grain motion is entirely perpendicular to field lines, we noted in the text that the ratio of Lorentz to drag forces is $\sim 0.1 T_{100 \mathrm{~K}} a_{\mu}^{-1} n_{10}^{-1 / 2}$. So while it is negligible for the largest grains in high-density regions (cores) that will actually form stars, it is by no means clear that we can completely ignore Lorentz forces (for a more detailed analytic comparison, see Yan et al. 2004). Unfortunately, the grain charge and the magnetic field strength are both highly uncertain under the conditions of interest; we therefore do not explicitly include Lorentz forces in our simulations. In future work, however, we intend to investigate this in more detail.

To a first approximation, in most studies of turbulence and star formation the full effects of cooling can be reasonably represented by simply adopting an isothermal $(\gamma=1)$ equation of state (see, e.g., Nordlund \& Padoan 1999; Ostriker et al. 1999; Li et al. 2005; Krumholz et al. 2007; Kritsuk et al. 2011; Veltchev et al. 2011; Konstandin et al. 2012; Molina et al. 2012; Hopkins 2013c). We do so here. Although cooling physics may be more complicated and less efficient under highredshift conditions, the isothermal approximation is still reasonable; moreover, we considered the opposite regime of inefficient cooling and ran two simulations with $\gamma=7 / 5$ and $\gamma=5 / 3$, respectively. Although the structures that form in the gas are modified (as the gas is less compressible), the dust-togas ratio fluctuations are qualitatively identical (presumably 
because they are driven by the vorticity field, not by compressible gas motions). In fact, the maximum dust-to-gas ratio fluctuations have slightly larger magnitude in these adiabatic cases.

Obviously, for the sake of computational expense and resolution, we simplify by restricting to $2 \mathrm{D}$. However, in P. F. Hopkins \& H. Lee (2015, in preparation), we consider a suite of both 2D and 3D simulations (lower-resolution and without shear) and show that all the same conclusions apply in 3D (for the same $\tau_{\mathrm{s}}$ ), albeit usually with a slightly weaker maximum grain clustering amplitude.

These simulations are intended to qualitatively illustrate dust dynamics. We therefore do not attempt to follow cosmological galaxy and/or star formation, or their enrichment of the medium and/or dust formation, but simply trace a pre-existing grain population in a turbulent disk.

\section{A.3. Initial Conditions}

Without loss of generality, we adopt code units where $\left\langle c_{s}\right\rangle=1,\left\langle\rho_{\mathrm{g}}\right\rangle=1$, and $\Omega=1$ (at the box center). The physics of our problem is then completely specified by three dimensionless parameters: the Mach number $\mathcal{M}$ of the (driven) turbulence, the dimensionless average stopping time $\left\langle\tau_{\mathrm{s}}\right\rangle \equiv$ $\bar{\rho}_{\text {solid }} a \Omega /\left(\left\langle\rho_{\mathrm{g}}\right\rangle\left\langle c_{s}\right\rangle\right)$, and the size of the box in code units $L /\left(c_{s} / \Omega\right)$. We always choose the driving scale of the turbulence to correspond to a narrow range in Fourier space (factor $\sim 2$ in $k$ ) centered on the disk scale height $H=\sigma / \Omega=\left(c_{s} / \Omega\right)\left(1+\mathcal{M}^{2}\right)^{1 / 2}$. We adopt an isothermal equation of state (see above), and $q=1$, appropriate for a galactic disk with $V_{c}=$ constant. Both gas and dust are initialized with uniform density.

Motivated by cosmological simulations of these first galaxies, in this paper we focus on transonic turbulence with 1D Mach numbers $\mathcal{M} \sim 1-2$, and motivated by observed physical grain sizes, we consider $\tau_{\mathrm{s}} \sim 0.01,0.1,1$. Finally, we perform all simulations at $1024^{2}$ resolution. We have considered numerical resolution studies (from $64^{2}$ to $2048^{2}$ ), and for the $\tau_{\mathrm{s}}$ which we simulate here, $1024^{2}$ gives wellconverged results (for smaller $\tau_{\mathrm{s}}$, however, grains will cluster on still smaller scales, so higher resolution is necessary). We consider two box sizes, with side-length $L=1$ in code units (i.e., $\left.L \approx H / \sqrt{1+\mathcal{M}^{2}}\right)$, and $L=5\left(L \approx 5 H / \sqrt{1+\mathcal{M}^{2}}\right)$. The former zooms in on scales we expect to contain $\sim 1$ massive GMC complex, while the latter contains many such complexes (but less well-resolved). Because we see good convergence, we analyze the larger boxes in the text since the gas statistics are better sampled, but the results are similar in either case.

We seed the simulations with trace initial magnetic fields; these fields are amplified by the turbulent dynamo until they saturate around equipartition (magnetic energy about $\sim 5 \%$ of the kinetic energy, in good agreement with other simulations using a variety of different numerical techniques and analytic estimates; see Schekochihin et al. 2004; Brandenburg \& Subramanian 2005; Federrath et al. 2014). However, because they only indirectly influence the dynamics by weakly changing the structure of turbulence, we find that magnetic fields do not significantly change our conclusions compared to hydro-only runs.

All simulations are run for a timescale at least $\sim 20 \Omega^{-1}$. We discard the first five dynamical times, as the statistical properties might be biased by our initial conditions. After about $t \sim \Omega^{-1}$, the galaxy reaches steady-state and we see no systematic variation in the Mach numbers, magnetic field strength, or distribution of dust/gas densities; we therefore simply average the statistical results over the retained time snapshots for each simulation. Finally, we analyze the results as in P. F. Hopkins \& H. Lee (2015, in preparation), using a local kernel density estimator to measure the distribution of dust and gas densities at all points in the simulation.

\section{References}

Abate, C., Pols, O. R., Stancliffe, R. J., et al. 2015, A\&A, 581, A62 Abergel, A., Bernard, J. P., Boulanger, F., et al. 2002, A\&A, 389, 239

Altobelli, N., Dikarev, V., Kempf, S., et al. 2007, JGRA, 112, 7105

Aoki, W., Beers, T. C., Lee, Y. S., et al. 2013, AJ, 145, 13

Aoki, W., Norris, J. E., Ryan, S. G., Beers, T. C., \& Ando, H. 2002, ApJL, 576, L141

Bai, X.-N., \& Stone, J. M. 2010a, ApJ, 722, 1437

Bai, X.-N., \& Stone, J. M. 2010b, ApJS, 190, 297

Bai, X.-N., \& Stone, J. M. 2010c, ApJL, 722, L220

Barkana, R., \& Loeb, A. 2001, PhR, 349, 125

Bauer, A., \& Springel, V. 2012, MNRAS, 423, 3102

Bec, J., Biferale, L., Cencini, M., Lanotte, A. S., \& Toschi, F. 2009, arXiv:0905.1192

Bianchi, S., \& Schneider, R. 2007, MNRAS, 378, 973

Bonifacio, P., Caffau, E., Spite, M., et al. 2015, A\&A, 579, A28

Bracco, A., Chavanis, P. H., Provenzale, A., \& Spiegel, E. A. 1999, PhFl, 11,2280

Brandenburg, A., \& Subramanian, K. 2005, PhR, 417, 1

Carballido, A., Stone, J. M., \& Turner, N. J. 2008, MNRAS, 386, 145

Carollo, D., Freeman, K., Beers, T. C., et al. 2014, ApJ, 788, 180

Chen, B., Dai, X., Kochanek, C. S., \& Chartas, G. 2013, arXiv:1306.0008

Cherchneff, I., \& Dwek, E. 2010, ApJ, 713, 1

Chiaki, G., Schneider, R., Nozawa, T., et al. 2014, MNRAS, 439, 3121

Chiaki, G., \& Yoshida, N. 2015, MNRAS, 451, 3955

Chiar, J. E., Tielens, A. G. G. M., Whittet, D. C. B., et al. 2000, ApJ, 537, 749

Costantini, E., Freyberg, M. J., \& Predehl, P. 2005, A\&A, 444, 187

Cucchiara, A., Levan, A. J., Fox, D. B., et al. 2011, ApJ, 736, 7

Cuzzi, J. N., Hogan, R. C., Paque, J. M., \& Dobrovolskis, A. R. 2001, ApJ, 546,496

de Bennassuti, M., Schneider, R., Valiante, R., \& Salvadori, S. 2014, MNRAS, 445, 3039

De Cia, A., Ledoux, C., Savaglio, S., Schady, P., \& Vreeswijk, P. M. 2013, A\&A, 560, A88

De Marchi, G., \& Panagia, N. 2014, MNRAS, 445, 93

Dittrich, K., Klahr, H., \& Johansen, A. 2013, ApJ, 763, 117

Dopcke, G., Glover, S. C. O., Clark, P. C., \& Klessen, R. S. 2013, ApJ, 766, 103

Draine, B. T. 2003, ARA\&A, 41, 241

Draine, B. T., \& Li, A. 2007, ApJ, 657, 810

Draine, B. T., \& Salpeter, E. E. 1979, ApJ, 231, 77

Draine, B. T., \& Sutin, B. 1987, ApJ, 320, 803

Dwek, E., Staguhn, J., Arendt, R. G., et al. 2014, ApJL, 788, L30

Elmegreen, B. G. 1979, ApJ, 232, 729

Federrath, C., Klessen, R. S., \& Schmidt, W. 2008, ApJL, 688, L79

Federrath, C., Schober, J., Bovino, S., \& Schleicher, D. R. G. 2014, ApJL, 797, L19

Flagey, N., Noriega-Crespo, A., Boulanger, F., et al. 2009, ApJ, 701, 1450

Frebel, A. 2010, AN, 331, 474

Frebel, A., Chiti, A., Ji, A. P., Jacobson, H. R., \& Placco, V. M. 2015, ApJL, 810, L27

Frebel, A., Simon, J. D., \& Kirby, E. N. 2014, ApJ, 786, 74

Gall, C., Hjorth, J., Watson, D., et al. 2014, Natur, 511, 326

Gerakines, P. A., Whittet, D. C. B., Ehrenfreund, P., et al. 1999, ApJ, 522, 357

Gibb, E. L., Whittet, D. C. B., Schutte, W. A., et al. 2000, ApJ, 536, 347

Gilmore, G., Norris, J. E., Monaco, L., et al. 2013, ApJ, 763, 61

Goldsmith, P. F., Heyer, M., Narayanan, G., et al. 2008, ApJ, 680, 428

Gordon, K. D., Clayton, G. C., Misselt, K. A., Landolt, A. U., \& Wolff, M. J. 2003, ApJ, 594, 279

Greif, T. H., Johnson, J. L., Klessen, R. S., \& Bromm, V. 2008, MNRAS, 387, 1021

Grun, E., Zook, H. A., Baguhl, M., et al. 1993, Natur, 362, 428

Guan, X., \& Gammie, C. F. 2008, ApJS, 174, 145

Hansen, T., Hansen, C. J., Christlieb, N., et al. 2015, ApJ, 807, 173 
Heger, A., \& Woosley, S. E. 2010, ApJ, 724, 341

Hendrix, T., \& Keppens, R. 2014, A\&A, 562, A114

Hirashita, H. 2010, MNRAS, 407, L49

Hirashita, H. 2012, MSAIS, 21, 131

Hirashita, H., Ferrara, A., Dayal, P., \& Ouchi, M. 2014, MNRAS, 443, 1704

Hirashita, H., \& Yan, H. 2009, MNRAS, 394, 1061

Hogan, R. C., \& Cuzzi, J. N. 2007, PhRvE, 75, 056305

Hogan, R. C., Cuzzi, J. N., \& Dobrovolskis, A. R. 1999, PhRvE, 60, 1674

Hopkins, P. F. 2013a, MNRAS, 428, 2840

Hopkins, P. F. 2013b, MNRAS, 430, 1653

Hopkins, P. F. 2013c, MNRAS, 430, 1880

Hopkins, P. F. 2014, ApJ, 797, 59

Hopkins, P. F. 2015, MNRAS, 450, 53

Hopkins, P. F. 2016a, MNRAS, 455, 89

Hopkins, P. F. 2016b, MNRAS, 456, 2383

Hopkins, P. F., \& Lee, H. 2016, MNRAS, 456, 4174

Hopkins, P. F., \& Raives, M. J. 2016, MNRAS, 455, 51

Hopkins, P. F., Strauss, M. A., Hall, P. B., et al. 2004, AJ, 128, 1112

Ishigaki, M. N., Tominaga, N., Kobayashi, C., \& Nomoto, K. 2014, ApJL, 792, L32

Jalali, M. A. 2013, ApJ, 772, 75

Jenkins, E. B. 2009, ApJ, 700, 1299

Ji, A. P., Frebel, A., \& Bromm, V. 2014, ApJ, 782, 95

Johansen, A., \& Youdin, A. 2007, ApJ, 662, 627

Johansen, A., Youdin, A., \& Mac Low, M.-M. 2009, ApJL, 704, L75

Jones, A. P., Tielens, A. G. G. M., \& Hollenbach, D. J. 1996, ApJ, 469, 740

Jones, A. P., Tielens, A. G. G. M., Hollenbach, D. J., \& McKee, C. F. 1994, ApJ, 433, 797

Keane, J. V., Tielens, A. G. G. M., Boogert, A. C. A., Schutte, W. A., \& Whittet, D. C. B. 2001, A\&A, 376, 254

Kemper, F., Vriend, W. J., \& Tielens, A. G. G. M. 2004, ApJ, 609, 826

Klessen, R. S., Glover, S. C. O., \& Clark, P. C. 2012, MNRAS, 421, 3217

Kochanek, C. S. 2014, MNRAS, 444, 2043

Konstandin, L., Girichidis, P., Federrath, C., \& Klessen, R. S. 2012, ApJ, 761, 149

Kritsuk, A. G., Nordlund, Å., Collins, D., et al. 2011, ApJ, 737, 13

Krumholz, M. R., Klein, R. I., \& McKee, C. F. 2007, ApJ, 656, 959

Kuo, T.-M., Hirashita, H., \& Zafar, T. 2013, MNRAS, 436, 1238

Lai, D. K., Rockosi, C. M., Bolte, M., et al. 2009, ApJL, 697, L63

Landgraf, M., Baggaley, W. J., Grün, E., Krüger, H., \& Linkert, G. 2000, JGR, 105,10343

Lee, Y. S., Beers, T. C., Masseron, T., et al. 2013, AJ, 146, 132

Lee, Y. S., Suda, T., Beers, T. C., \& Stancliffe, R. J. 2014, ApJ, 788, 131

Li, A., \& Draine, B. T. 2001, ApJ, 554, 778

Li, A., Liang, S. L., Kann, D. A., et al. 2008, ApJ, 685, 1046

Li, Y., Mac Low, M.-M., \& Klessen, R. S. 2005, ApJL, 620, L19

Maeder, A., Meynet, G., \& Chiappini, C. 2015, A\&A, 576, A56

Maiolino, R., Schneider, R., Oliva, E., et al. 2004, Natur, 431, 533

Marassi, S., Chiaki, G., Schneider, R., et al. 2014, ApJ, 794, 100

Marassi, S., Schneider, R., Limongi, M., et al. 2015, MNRAS, 454, 4250

Mathis, J. S., Rumpl, W., \& Nordsieck, K. H. 1977, ApJ, 217, 425

Mattsson, L., De Cia, A., Andersen, A. C., \& Zafar, T. 2014, MNRAS, 440, 1562

Meece, G. R., Smith, B. D., \& O'Shea, B. W. 2014, ApJ, 783, 75

Miville-Deschênes, M.-A., Boulanger, F., Joncas, G., \& Falgarone, E. 2002, A\&A, 381, 209

Molina, F. Z., Glover, S. C. O., Federrath, C., \& Klessen, R. S. 2012, MNRAS, 423, 2680

Molster, F. J., Waters, L. B. F. M., \& Tielens, A. G. G. M. 2002a, A\&A, 382,222

Molster, F. J., Waters, L. B. F. M., Tielens, A. G. G. M., Koike, C., \& Chihara, H. 2002b, A\&A, 382, 241

Monchaux, R., Bourgoin, M., \& Cartellier, A. 2010, PhFl, 22, 103304

Monchaux, R., Bourgoin, M., \& Cartellier, A. 2012, IJMF, 40, 1

Muratov, A. L., Gnedin, O. Y., Gnedin, N. Y., \& Zemp, M. 2013, ApJ, 772,106
Nomoto, K., Tominaga, N., Umeda, H., Kobayashi, C., \& Maeda, K. 2006, NuPhA, 777, 424

Nordlund, Å. K., \& Padoan, P. 1999, in Interstellar Turbulence, ed. J. Franco \& A. Carraminana (Cambridge: Cambridge Univ. Press), 218

Norris, J. E., Gilmore, G., Wyse, R. F. G., Yong, D., \& Frebel, A. 2010a, ApJL, 722, L104

Norris, J. E., Yong, D., Bessell, M. S., et al. 2013, ApJ, 762, 28

Norris, J. E., Yong, D., Gilmore, G., \& Wyse, R. F. G. 2010b, ApJ, 711, 350

Nozawa, T., Kozasa, T., Habe, A., et al. 2007, ApJ, 666, 955

Nozawa, T., Kozasa, T., \& Nomoto, K. 2012, ApJL, 756, L35

Nozawa, T., Yoon, S.-C., Maeda, K., et al. 2014, ApJL, 787, L17

Nyland, K., Alatalo, K., Wrobel, J. M., et al. 2013, ApJ, 779, 173

Olla, P. 2010, PhRvE, 81, 016305

Omukai, K., Tsuribe, T., Schneider, R., \& Ferrara, A. 2005, ApJ, 626, 627

Ostriker, E. C., Gammie, C. F., \& Stone, J. M. 1999, ApJ, 513, 259

Padoan, P., Cambrésy, L., Juvela, M., et al. 2006, ApJ, 649, 807

Pan, L., Padoan, P., Scalo, J., Kritsuk, A. G., \& Norman, M. L. 2011, ApJ, 740, 6

Pawlik, A. H., Milosavljević, M., \& Bromm, V. 2013, ApJ, 767, 59

Pei, Y. C. 1992, ApJ, 395, 130

Perley, D. A., Bloom, J. S., Klein, C. R., et al. 2010, MNRAS, 406, 2473

Perley, D. A., Morgan, A. N., Updike, A., et al. 2011, AJ, 141, 36

Pineda, J. L., Goldsmith, P. F., Chapman, N., et al. 2010, ApJ, 721, 686

Placco, V. M., Frebel, A., Beers, T. C., \& Stancliffe, R. J. 2014, ApJ, 797, 21

Placco, V. M., Frebel, A., Lee, Y. S., et al. 2015, ApJ, 809, 136

Poppe, A., James, D., Jacobsmeyer, B., \& Horányi, M. 2010, GeoRL, 37, 11101

Price, D. J., \& Federrath, C. 2010, MNRAS, 406, 1659

Richards, G. T., Hall, P. B., Vanden Berk, D. E., et al. 2003, AJ, 126, 1131

Rouson, D. W. I., \& Eaton, J. K. 2001, JFM, 428, 149

Schady, P., Dwelly, T., Page, M. J., et al. 2012, A\&A, 537, A15

Schekochihin, A. A., Cowley, S. C., Taylor, S. F., Maron, J. L., \& McWilliams, J. C. 2004, ApJ, 612, 276

Schmidt, W., Federrath, C., \& Klessen, R. 2008, PhRvL, 101, 194505

Schnee, S., Mason, B., Di Francesco, J., et al. 2014, MNRAS, 444, 2303

Schneider, R., Ferrara, A., Salvaterra, R., Omukai, K., \& Bromm, V. 2003, Natur, 422, 869

Schneider, R., Omukai, K., Bianchi, S., \& Valiante, R. 2012a, MNRAS, 419, 1566

Schneider, R., Omukai, K., Inoue, A. K., \& Ferrara, A. 2006, MNRAS, 369,1437

Schneider, R., Omukai, K., Limongi, M., et al. 2012b, MNRAS, 423, L60

Sofia, U. J., \& Meyer, D. M. 2001, ApJL, 554, L221

Sparre, M., Hartoog, O. E., Krühler, T., et al. 2014, ApJ, 785, 150

Squires, K. D., \& Eaton, J. K. 1991, PhFl, 3, 1169

Takahashi, K., Umeda, H., \& Yoshida, T. 2014, ApJ, 794, 40

Thoraval, S., Boisse, P., \& Duvert, G. 1997, A\&A, 319, 948

Thoraval, S., Boissé, P., \& Duvert, G. 1999, A\&A, 351, 1051

Tielens, A. G. G. M., Waters, L. B. F. M., Molster, F. J., \& Justtanont, K. 1998, Ap\&SS, 255, 415

Tominaga, N., Iwamoto, N., \& Nomoto, K. 2014, ApJ, 785, 98

Tominaga, N., Umeda, H., \& Nomoto, K. 2007, ApJ, 660, 516

Umeda, H., \& Nomoto, K. 2005, ApJ, 619, 427

Updike, A. C., Hartmann, D. H., \& Kann, D. A. 2011, BAAS, 43, 108.01

Veltchev, T. V., Klessen, R. S., \& Clark, P. C. 2011, MNRAS, 411, 301

Weingartner, J. C., \& Draine, B. T. 2001, ApJ, 548, 296

Wise, J. H., \& Abel, T. 2008, ApJ, 685, 40

Wise, J. H., Turk, M. J., \& Abel, T. 2008, ApJ, 682, 745

Witt, A. N., Smith, R. K., \& Dwek, E. 2001, ApJL, 550, L201

Woosley, S. E., \& Weaver, T. A. 1995, ApJS, 101, 181

Yan, H., Lazarian, A., \& Draine, B. T. 2004, ApJ, 616, 895

Yong, D., Norris, J. E., Bessell, M. S., et al. 2013, ApJ, 762, 27

Yoshimoto, H., \& Goto, S. 2007, JFM, 577, 275

Youdin, A. N., \& Goodman, J. 2005, ApJ, 620, 459

Zafar, T., Watson, D., Elíasdóttir, Á., et al. 2012, ApJ, 753, 82

Zafar, T., \& Watson, D. 2013, A\&A, 560, A26 